\title{
CARTAGINE: LA CITTÀ E LE NECROPOLI \\ RAPPORTO PRELIMINARE SULLA PRIMA CAMPAGNA DI SCAVI (2021) DELL'INSTITUT NATIONAL DU PATRIMOINE E DELL'UNIVERSITÀ DI ROMA «LA SAPIENZA»
}

\author{
Lorenzo Nigro - Federico Cappella - Alfredo Coppa - Francesco Genchi \\ Sapienza University of Rome (Italie) \\ Moez Achour - Mounir Fantar \\ Institut National du Patrimoine (Tunisie)
}

Dans le cadre de la coopération scientifique entre l'Institut National du Patrimoine et l'Université de Rome "La Sapienza », une première campagne de fouille a eu lieu dans un secteur de la nécropole punique de Dermech à Carthage, dans l'enceinte du parc des thermes d'Antonin et aux abords de la colline de Borj-Jedid, du 17 octobre au 10 novembre 2021. Sous les vestiges romains et byzantins, neuf tombes - à fosse et à chambre unique avec puits d'accès vertical - creusées dans le roc, ont été dégagées dans un secteur datable du VIIème et Vème siècles av. J.-C.

Within the framework of the scientific cooperation agreement between the Institut National du Patrimoine and the University of Rome "La Sapienza», from 17 October to 10 November 2021 a first archaeological season of excavation took place in a sector of the Phoenician and Punic necropolis of Dermech in Carthage, within the park of the Antonin's thermal baths beside the Borj-Jedid hill,. Beneath the Roman and Byzantine remains, nine tombs - pit and single chamber with vertical shaftdug into the rock, have been unearthed in a sector datable to the $7^{\text {th }}$ and $5^{\text {th }}$ centuries $B C$.

Keywords: Punic tombs; Carthage; Phoenician; Antonine's Baths; Dermech

\section{INTRODUZIONE: LA MiSSIONE ITALO-TUNISINA A CARTAGINE}

Dal 17 ottobre al 10 novembre 2021 si è svolta la prima campagna (2021) di scavo, studio e valorizzazione a Cartagine da parte della missione congiunta italo-tunisina dell'Università di Roma «La Sapienza» e dell'Institut National du Patrimoine (INP) della Tunisia $^{1}$. La missione è stata costituita con una convenzione nell'ambito di un accordo

1 La Missione archeologica a Cartagine - Mission archéologique à Carthage - è diretta dal Dott. Mounir Fantar, Directeur du Département des Monuments et des Sites Antiques presso l'Institut National du Patrimoine della Tunisia e dal Prof. Lorenzo Nigro dell'Università di Roma «La Sapienza». Durante la prima campagna (2021), le indagini sul campo sono state coordinate dal Dott. Federico Cappella per l'Università di Roma «La Sapienza» e dal Dott. Moez Achour, Conservateur en chef du Musée National de Carthage e responsabile delle aree archeologiche dipendenti dal museo, tra le quali quella nell'area di Dermech oggetto delle ricerche. Responsabile scientifico degli studi antropologici è il Prof. Alfredo Coppa, docente onorario dell'Università «La Sapienza» di Roma. Di seguito l'elenco dei partecipanti - ricercatori, dottorandi, studenti e tecnici - che hanno preso parte alla Missione congiunta della Sapienza e dell'INP: Francesco Genchi (geomatico), Licia De Vito (disegnatrice), Francesco Caputo (archeologo), Martina De Giuseppe (fotografa), Flaminia Angelici (antropologa), Fahmi Kacem (archeologo), Maha Bannour (archeologa), Fatouma Boukhris e Shayma Dabboussi (archeologhe e disegnatrici sul campo). Allo scavo hanno inoltre partecipato cinque operai tunisini specializzati: Abdel Khader Razak, Ferjani Ben Amor, Mohammed Trabelsi, Mohammed Bou 
quadro di collaborazione tra i due enti, sotto l'egida del Ministero degli Affari Esteri e della Cooperazione italiano, al fine di condurre indagini archeologiche e antropologiche a Cartagine per contribuire alla ricostruzione storica e culturale dell'antica capitale del Mediterraneo.

Le ricerche sono state finanziate dall’Università di Roma «La Sapienza», dal Ministero degli Affari Esteri e della Cooperazione e dal Ministero dell'Università e Ricerca italiani e dall'Institut National du Patrimoine (INP) della Tunisia.

Le indagini della prima campagna a Cartagine hanno interessato la necropoli di Dermech nell'area compresa all'interno del complesso delle Terme di Antonino in un settore nel quale scavi precedenti avevano identificato tombe databili tra VII e VI secolo a.C., con l'intenzione di ampliare l'area oggetto di scavi e di scendere progressivamente fino agli strati più profondi dell'occupazione fenicia e punica.

\section{STORIA DEGLI SCAVI NELLA NECROPOLI DI DERMECH, CARTAGINE ${ }^{2}$}

L'identificazione della necropoli di Dermech nelle immediate adiacenze delle monumentali Terme di Antonino si deve agli scavi condotti nel 1862 da parte dell'ingegnere A. Gouvet che scoprì alcune tombe, già espilate in antico, scavando una trincea profonda $10 \mathrm{~m}$ subito a nord-ovest del complesso termale in corrispondenza delle cisterne romane ${ }^{3}$. Nel 1885 ventiquattro tombe di epoca fenicio-punica vennero rinvenute da Jean Vernaz nell'area delle terme durante gli scavi per la ricerca dell'acquedotto romano $^{4}$. A cavallo tra XIX e XX secolo, informazioni più consistenti provengono dagli scavi sistematici di Paul Gauckler condotti a partire dal 1899 in qualità di Directeur du Service archéologique de Tunisie che restituirono un numero eccezionale di tombe ${ }^{5}$. Dopo il rinvenimento di alcune sepolture nel corso degli scavi condotti da Louis Poinssot e Raymond Lantier negli anni '20 del Novecento per conto del Service des Antiquité ultimi scavi sistematici della necropoli di Dermech nell'area delle Terme di Antonino si ebbero ad opera di Pierre Cintas nel $1942^{7}$ e, infine, di Maurice Vezat, tra il 1950 e il 1953 , questi ultimi, tuttavia, rimasti inediti.

Nonostante la necropoli sia stata esplorata a partire dalla seconda metà dell' 800 , come ebbe modo di sperimentare a suo tempo Hélène Benichou-Safar, alla quale si deve la più completa disamina delle necropoli di Cartagine $^{8}$, la principale problematica nella

Laress e Ahmed Amine. I §§ 1, 2, 3 sono di L. Nigro, M. Fantar, M. Achour; il § 4 di F. Genchi; il 55 di F. Cappella; il § 6 di L. Nigro, A. Coppa.

2 Una sintesi della storia degli scavi delle necropoli di Cartagine è in Benichou-Safar 1982, 19-32 e in particolare 20-23 sugli scavi alla necropoli di Dermech.

Daux 1869, 54-55.

Vernaz 1887, 151-164.

Una sintesi degli scavi e delle scoperte sono rintracciabili nel volume postumo Nécropoles Punques de Carthage del 1915: «C'est là, à mon avis, que les premiers Phéniciens ont débarqué pour prendre possession du sol de Carthage; c'est sur la plage de Borj-Djedid, à Dermech, qu'ils ont installé leurs premières demeures, et tout près de là, leurs premiers tombeaux» (Gauckler 1915, 380).

Poinssot - Lantier 1927, 437-474.

Cintas 1976, 330-332.

Benichou-Safar 1982. Sulla topografia di Cartagine arcaica si veda anche Maraoui-Telmini - Chelbi - Docter 2013. 
ricostruzione della storia degli studi e degli scavi delle necropoli puniche della colonia fenicia risiede proprio nel numero di interventi condotti nel tempo senza un progetto unitario, nella carenza o addirittura nell'assenza di dati dai rapporti di scavo e, talvolta, nella stessa metodologia inadeguata ${ }^{9}$.

Per quanto riguarda l'area della necropoli di Dermech sottoposta nuovamente ad indagini archeologiche da parte dalla neonata missione italo-tunisina, questa si trova all'interno del complesso delle Terme di Antonino e corrisponde alla cosiddetta Zone fouilles Vezat $1950^{10}$ (fig. 1). Dopo la Seconda Guerra Mondiale, durante il periodo del Protettorato francese, quest'area divenne infatti oggetto di scavi archeologici condotti da M. Vezat, nominato professore a Tunisi nel 1948, sotto la supervisione di P. Cintas e G.-Ch. Picard, all'epoca rispettivamente il responsabile del Département des Antiquités puniques e il Directeur des Antiquités de Tunisie. Gli scavi della Mission archéologique française del 1950, tuttavia, sono ad oggi inediti e le prime e scarne informazioni a disposizione si possono ricavare dal "Report sur l'archéologie romaine en Tunisie dans le premier semestre 1950” di G.-Ch. Picard, edito nel 1952, nel quale si riporta una labile testimonianza della presenza di alcune tombe a fossa di epoca punica senza cenno ai materiali rinvenuti o all'estensione effettiva dello scavo". Segue nel 1969 un contributo dal titolo "Fouilles inédites. Comment Carthage enterrait ses morts aux VIè et Vè siècles" pubblicato dallo stesso M. Vezat nel quale vengono menzionate diciotto tombe scavate tra il 1950 e il 1953 nell'area di Dermech, ma per le quali, anche in questo caso, non vengono fornite indicazioni puntuali ${ }^{12}$.

Con le indagini di M. Vezat si pone fine agli scavi della necropoli di Dermech a Cartagine fino all'anno della prima campagna della missione congiunta italo-tunisina della Sapienza e dell'INP dell'ottobre del 2021.

\section{LOCALIZZAZIONE}

La prima campagna (2021) della Missione congiunta dell’Università di Roma «La Sapienza» e dell'INP a Cartagine è stata indirizzata alla esplorazione preliminare di un settore della necropoli di Dermech localizzato all'interno della più vasta area del parco archeologico delle Terme di Antonino sulle pendici meridionali della collina di BorjDjedid. Il parco si estende su una vasta area compresa tra l'Avenue Habib Bourguiba ad ovest, l'Avenue des Thermes a sud e la residenza presidenziale a nord. Tra i monumenti all'interno del parco, prime per magnificenza sono le terme pubbliche del II secolo d.C. realizzate per volere dell'imperatore Antonino Pio (138-161 d.C.) tra il 157/159 e il 162 d.C. ${ }^{13}$. Queste, dopo la distruzione di Cartagine del 146 a.C., rappresentano uno dei più grandi e ambiziosi progetti pubblici a carattere monumentale della nuova Cartagine

\footnotetext{
Benichou-Safar 1982, 10, 16-18.

Benichou-Safar 1982, 34, fouille n. 2 e fig. 3:2

«Diverses tombes á puits s'échelonnant de la fin du VII au début du VIe siècle ont été fouillées par M. Vezat. Au niveau romain ne subsistent que des éléments épars» (Picard 1952, 155).

12 Vezat 1969,67 , nota 1,154

13 L'attribuzione delle terme al periodo dell'Imperatore Antonino Pio è fondata sul ritrovamento di un'iscrizione latina (CIL VIII, 12513). Al riguardo si rimanda a Aounallah - Baklouti - Mosca 2018, 284.
} 
romana $^{14}$ : con un'estensione di $17.850 \mathrm{~m}^{2}$ sono il complesso termale più esteso delle province romane d'Africa finora scoperto ${ }^{15}$. Il fabbricato monumentale, nonostante il rapido declino iniziato almeno a partire dagli inizi del V secolo d.C. parallelamente alla conquista vandala del 439 d.C. ${ }^{16}$, resterà in uso in età bizantina fino al VI-VII secolo d.C.

Insieme alle rovine di età imperiale e del periodo bizantino ${ }^{17}$, all'interno del parco delle Terme di Antonino sono documentati anche i resti della più antica città punica come una fornace a pianta circolare, ancora visibile nei pressi della basilica di Dermech II, o come le diverse tombe - a fossa, a camera o costruite - distribuite all'interno del parco e databili tra VIII e V secolo a.C.

L'area della necropoli fenicia e punica indagata dalla Missione è localizzata nel tratto nord-occidentale dell'insula 7, compresa tra il Cardo XVI e il Decumano IV dell'antica centuriazione romana (fig. 1). Quest'area, denominata "Dermech - Nécropole", è stata a sua volta preliminarmente suddivisa in due settori, approssimativamente quadrangolari, denominati Settore A, a nord, e Settore B, a sud.

\section{TOPOGRAFIA}

Il Settore A misura 11,5 × 13,2 × $10 \times 12,5 \mathrm{~m}$ con un'estensione di 137,7 $\mathrm{m}^{2}$ (fig. 2), si trova tra i 9 e 7 m s.l.m. (in lieve pendenza da nord a sud) ed è localizzabile alle coordinate geografiche $36^{\circ} 51^{\prime} 17.95^{\prime} \mathrm{N}, 10^{\circ} 20^{\prime} 1.00^{\prime \prime} \mathrm{E}$. Si tratta di un appezzamento delimitato a ovest dal muro moderno che fiancheggia e delimita il viale turistico in asse con l'ingresso principale del parco delle Terme di Antonino che ha mantenuto l'andamento del Cardo XVI; a nord, da un muro perpendicolare, sulla falsa riga del precedente, in asse con il Decumano IV (che ricalca l'andamento di un grande muro d'età bizantina: M.1); ad est, dal muro di una struttura rettangolare (M.3) rimaneggiata in età bizantina, forse una cappella, divisa in anticamera e vestibolo con ingresso a cinque gradini posizionato nell'angolo meridionale della parete orientale ${ }^{18}$; a sud, da una struttura muraria con andamento est-

14 Dopo il tentativo fallimentare di Caio Gracco nel 122 a.C. di dedurre alcuni coloni sulla ormai distrutta Cartagine punica, la colonia, che prenderà il nome di Colonia Iulia Concordia Carthago, venne fondata sotto Augusto nell'anno 29 d.C. (Aounallah 2018, 238-243).

15 Aounallah - Baklouti - Mosca 2018, 286.

16 È in questo momento che è stato ipotizzato il crollo della copertura del frigidarium delle Terme: Baratte Béjaoui 2018, 376.

17 Si vedano ad esempio la c.d. Cappella di Asterio e le due basiliche Dermech I e II (Alexander - Ben Abed-Ben Khader - Guy 1996, 361-362; Baratte - Béjaoui 2018, 385-387).

18 Il piccolo edificio, sulla cui interpretazione funzionale e cronologica restano ancora dei dubbi (Ben Abed-Ben Khader 1999, 39), è orientato NNE-SSO, aveva le pareti originariamente intonacate di bianco e presenta lastricati di marmo (opus scutulatum) alternati a mosaici con decorazioni essenziali (opus tessellatum): a semplici tessere bianche nell'anticamera e a motivo floreale stilizzato su fondo bianco nel vestibolo. A quest'ultimo, il cui piano si trova ad una quota inferiore rispetto all'anticamera, si accedeva mediante una scalinata di due bassi gradini di marmo. Una descrizione puntuale della decorazione musiva è in Ben AbedBen Khader 1999, 39, pianta 5, n. 66. La struttura, per la quale sono distinguibili diverse fasi costruttive, vede il rimpiego di alcuni elementi architettonici più antichi. Nel vestibolo con falsa copertura a volta crociata, al centro del lato occidentale, è presente un blocco di pietra modanato che trova alcuni confronti con le cornici poste all'ingresso degli ipogei punici (es: Cintas 1970, tav. LX:5; Benichou-Safar 1982, 113-115, in particolare fig. 60) mentre nel lato breve a sud si trova un blocco di marmo bianco, un architrave, con iscrizione in latino rivolta verso l'ingresso nella quale si legge "iussu Domini Aescu[lapii]". Purtroppo, il 
ovest (M.5) ancora parzialmente interrata che si imposta direttamente al di sopra della copertura monumentale di una tomba costruita del VI secolo a.C. (T.V).

Nel cuore dell'area, a pochi centimetri di profondità, emerge la tenera roccia vergine entro la quale furono ricavate durante il periodo arcaico (VII-VI secolo a.C.) alcune tombe del tipo a fossa e altre del tipo a camera con pozzetto d'accesso laterale. Durante la prima campagna (2021), nel Settore A sono state identificate nove tombe, cinque delle quali sono state completamente scavate (T. I, II, III, VI, VIII). Sei tombe presentano un orientamento E-O (T. I, II, III, IV, VII, VIII), mentre tre sono orientate diversamente T.V, T.VI e T.IX. Sul lato settentrionale, al di sopra delle tombe fenicie insistono strutture murarie di età imperiale (M.31, M.37) e bizantina (M.1, B.11), cui devono essere riferite alcune istallazioni, come la cisterna C.13 ricavata sfruttando lo spazio della T. IV, che hanno compromesso già in antico l'integrità della necropoli.

La seconda area di scavo, il Settore B, è un saggio di $5 \times 5 \mathrm{~m}$ collocato $10 \mathrm{~m}$ a sud del Settore A, il cui limite occidentale è rappresentato dallo stesso muro moderno che fiancheggia e delimitala il viale turistico in asse con il Cardo XVI. Il saggio nel Settore B è stato aperto al fine d'indagare l'estensione della necropoli verso sud e studiare la stratigrafia dei livelli superiori, solo parzialmente rintracciabile nel Settore A. Differentemente da quanto osservato nel Settore A, però, in cui la roccia vergine nei punti più alti si trova a pochi centimetri al di sotto degli strati di dilavamento e accumulo naturale, nel Settore $B$, a causa della pendenza del terreno, questa si trova ad una maggiore profondità e sono presenti pertanto diversi livelli d'occupazione.

\section{STRATIGRAFIA, CRONOLOGIA E RITROVAMENTI}

\subsection{Settore A: tipologia delle tombe della necropoli fenicia}

Venendo alla disamina delle tombe individuate all'interno del Settore A, è necessario precisare che queste, al momento dello scavo, sono state trovate per la maggior parte colme solamente di terreno di riporto o di dilavamento. Non è dunque possibile avere informazioni dirette sul corredo funerario - salvo rari residui sporadici - né, tantomeno, stabilire con certezza quale tipo di sepoltura ospitassero, ovvero sapere se le stesse tombe

blocco è spezzato e il resto dell'iscrizione indecifrabile. Sempre a Cartagine, presso la collina del Teatro, è stata rinvenuta negli anni '40 del Novecento un'iscrizione identica su architrave (AE, 1949, 56. Si veda sul ritrovamento Picard 1949, 507-511 che attribuisce il blocco ad una struttura circolare che identifica come sacello di Esculapio e la diversa interpretazione quale martyrion del IV secolo d.C. offerta da Lancel 1989, 656. Per un quadro generale si rimanda a Cadotte 2007, 188, 523, n.185. Di questa non si dispone della documentazione fotografica e grafica e non può quindi escludersi che si tratti della stessa iscrizione oggi all'interno delle Terme di Antonino. Nonostante il ragionevole dubbio, il reperto resta di straordinario interesse in considerazione della nota assimilazione del dio fenicio Eshmoun ( ‘̌mn) con Esculapio (sull'argomento si veda Cadotte 2007, 165-169, con bibliografia di riferimento) e ben si inserirebbe nel panorama delle iscrizioni dedicate ad Esculapio da ambienti termali dell'Africa romana. Significativi in questo senso sono i ritrovamenti da Leptis Magna (AE, 1925, 105), da Timgad (CIL, VIII, 2340) e soprattutto dalle Terme d'Estate di Thuburbo Maius dove su un'iscrizione, originariamente disposta all'interno del tempio d'Esculapio, si legge l'obbligo di astenersi per tre giorni da rapporti sessuali, dal consumo di fagioli e di carne di maiale e, significativo in virtù del contesto di ritrovamento, dal frequentare i bagni (ILAf, 225; AE, 1916,112. Vattioni 1978, 13-31; Kleijwegt 1994; Cadotte 2007, 185). 
fossero state riutilizzate anche in età punica ${ }^{19}$. Tuttavia, sulla base dei dati recuperati durante la prima campagna e dei confronti con le altre aree funerarie di Cartagine, è possibile riflettere sugli aspetti tipologici e rituali, ricostruendo, almeno in parte, la storia e lo sviluppo della necropoli di Dermech in quest'area.

L'assenza dei corredi e delle sepolture è legata a due fattori principali: 1) l'area in questione venne sottoposta a scavi archeologici da parte di M. Vezat nei primi anni '50; 2) nel Settore A la roccia vergine, a causa della pendenza naturale verso sud, affiora spontaneamente dal terreno e doveva essere ancora parzialmente visibile al momento della costruzione degli edifici d'età romana imperiale e bizantina, che si sono appoggiati, talvolta rasandola ovvero scavandola, direttamente sull'area funeraria. Senza dubbio la presenza di tombe a vista in superficie è stata una delle cause principali del loro spoglio in antico ${ }^{20}$.

Sulla base degli scavi condotti su cinque tombe è stato possibile distinguere tre tipologie. Il primo tipo, al momento il più frequente, è costituito dalle tombe a fossa con aggetti laterali sui quattro lati ${ }^{21}$ (T.I, T.II, T.VIII, fig. 7) di forma rettangolare e con orientamento E-O. Si tratta di tombe in cui veniva deposto un singolo individuo inumato posto direttamente sulla roccia ${ }^{22} \mathrm{o}$ - più raramente - all'interno di un sarcofago ligneo con la testa rivolta ad est ${ }^{23}$. Il corredo funerario è modesto e non è composto da più di due vasi che nella maggior parte dei casi sono di produzione locale ${ }^{24}$. Le tombe di questo tipo sono una variazione cartaginese delle più diffuse tombe a fossa semplice documentate comunemente nelle necropoli del Mediterraneo centro-occidentale: l'espediente architettonico è utilizzato per sostenere le lastre di copertura della tomba attraverso la risega nel taglio della fossa ${ }^{25}$. Prova di questo impiego è la grande lastra di arenaria M.45 $(0,2 \times$ $0,5 \times 0,7 \mathrm{~m})$ divelta in antico e ritrovata all'interno della T.VIII addossata alla parete sudorientale, al di sopra del gradino sul lato meridionale (fig. 3). Le tombe hanno misure abbastanza regolari (circa 2,20 × 1,0 m) ${ }^{26}$ mentre la profondità complessiva varia dai 2,74 $\mathrm{m}$ della T.VIII ai 2,08 $\mathrm{m}$ e $1,32 \mathrm{~m}$ rispettivamente delle tombe T.I e T.II, seguendo la

19 Come nel caso di alcune tombe della collina di Byrsa (Delattre 1896, 31, 77-79).

20 D'altra parte, la sovrimposizione di monumenti romani e successivi sulle tombe fenicie era già stata osservata per la necropoli di Dermech durante gli scavi di P. Gauckler (1915, tavv. XIII, XIV, LIII) come documentato è lo spoglio delle tombe per far posto alle suddette strutture (Vernaz 1887, 154).

21 Tali aggetti misurano pochi centimetri, dai 5 ai $20 \mathrm{~cm}$.

22 «Dans ces fosses, réservé á un seul occupant. Les morts sont toujours inhumés, allongés sur le dos» (Cintas 1976, 267).

23 Sull'orientamento dei corpi nelle tombe di Dermech si rimanda a De Jonghe 2016, 107, in particolare nota 51. Per quanto riguarda il rituale funerario bisogna notare che, sebbene a Cartagine siano documentate sepolture a incinerazione, già dalla fine dell'VIII secolo a.C. è più frequente il rituale dell'inumazione (Bénichou-Safar 1982, 237-248 e 248-288), seguendo una tendenza opposta rispetto a quella che si registra negli altri centri fenici del Mediterraneo centrale ed occidentale (Bartoloni 2000, 70-71)

24 Cintas 1976, 268

25 De Jonghe 2016, 104 e tav. 1, tipo 2. Sul tipo si veda anche Bénichou-Safar 1982, 96-98, 348 e fig. 47, tipo VI.I, B e tipo VI.II. Tombe con i quattro aggetti laterali sono documentate nella regione africana del Capo Bon (es. Bartoloni 1973, 24, n. 3. Sull'architettura funeraria in questa regione si rimanda a Fantar 2002) e con minore frequenza anche in Sardegna dalle necropoli di Tharros (Fariselli 2006, 311-314, Tomba 5. Sulle tipologie tombali a Tharros si veda anche Secci 2016) e Monte Sirai (Bartoloni 1985, 254, Tomba. 47; Botto 2008, 1627, figg. 4-5, Tomba 164; Guirguis 2010, 105-112, Tomba 248; 142-144, Tomba 266).

26 Tomba I $(2,30 \times 1,16 \mathrm{~m})$; Tomba II $(2,18 \times 1,07 \mathrm{~m})$; Tomba VIII $(2,12 \times 0,96 \mathrm{~m})$ 
pendenza naturale della roccia entro la quale sono ricavate. D'altra parte, deve ritenersi probabile che non si tratti della profondità originale in quanto gli interventi di età romana e successiva hanno livellato parte del piano roccioso. Si mantiene invece costante la profondità della tomba calcolata dalla risega degli aggetti laterali, che è compresa tra 1,00 e $1,20 \mathrm{~m}^{27}$.

Proseguendo nella disamina delle tombe a fossa, se la T.I e la T.II presentano delle caratteristiche molto simili, alcune differenze sono ravvisabili nella T.VIII. In questa, al centro del lato settentrionale, è una rientranza irregolare, simile ad una nicchia, all'interno della quale era deposto un singolo reperto ceramico spezzato, un'ampia coppa carenata modellata e brunita a mano e ingobbiata interamente di rosso (fig. 11:1) ${ }^{28}$. A questo elemento si aggiunge la coppia di pedarole di forma approssimativamente semilunata, aventi diametro $0,20 \mathrm{~m}$, disposte specularmente nei lati lunghi per consentire una discesa più agevole all'interno della tomba, che non sono invece documentate nelle T.I e T.II. Tale assenza, tuttavia, potrebbe essere dovuta alla mancata conservazione della porzione superiore delle pareti delle tombe. Dal punto di vista cronologico, queste tombe di fattura semplice e poco elaborata possono essere considerate, sulla base delle caratteristiche architettoniche e tipologiche, le più antiche all'interno della necropoli di Dermech e sono ascrivibili al pieno VII secolo a.C. ${ }^{29}$

Alle spalle della T.I e della T.II, sul versante orientale, sono disposte parallelamente con medesimo orientamento E-O le T.III e T.IV. Se per quest'ultima non è possibile fornire informazioni di dettaglio, in quanto gravemente compromessa nella metà occidentale dallo sbancamento compiuto per la realizzazione della cisterna intonacata C.13, è possibile invece riflettere sulla planimetria della T.III (fig. 8:1), che mostra delle caratteristiche che la allontanano sensibilmente dalle tombe a fossa descritte precedentemente e costituisce un tipo ben definito: si tratta di una tomba a camera ipogea con pozzo d'accesso laterale corrispondente al "Type IX.1" della Benichou-Safar ${ }^{30}$. L'accesso laterale, situato ad ovest, è semi-rettangolare con orientamento e misure perfettamente conformi a quelle delle tombe a fossa $(2,25 \times 1,05 \mathrm{~m})$. Il piano del pozzetto, in pendenza verso est, conduce alla camera sepolcrale (L.130) attraverso un'apertura rettangolare (F.12) di 0,65 $\times 1,00 \mathrm{~m}$ posizionata al centro del lato est. Uno stretto corridoio (F.90), largo 0,65 m e articolato in una serie di due bassi e ampi gradini scoscesi, dà accesso alla camera ipogea che si trova ad una quota inferiore. Sul fondo del pozzetto sono alcune pietre di medie dimensioni probabilmente appartenute al muro di chiusura della camera ${ }^{31}$. Questa, intercettata e tagliata dalla struttura

Il volume medio dello spazio dove il defunto era deposto corrisponde a circa $1,3 / 1,5 \mathrm{~m}^{3}$.

CA.21.106/1.

9 Sulla cronologia e sui confronti per queste tombe si rimanda a Bénichou-Safar 1982, 348-349. In questo caso, le speculazioni sulla profondità delle tombe per determinarne la cronologia non sono possibili a causa degli interventi di livellamento che hanno interessato l'area a partire dal periodo romano.

30 Bénichou-Safar 1982, 105-106. Sul tipo si veda anche Cintas 1976, 272-273, fig. 27.

31 A Dermech, così come in altre aree di necropoli cartaginesi (Sainte-Monique, Ancona, Ard-et-Touibi, Dahar el Morali), è frequente il ricorso alla chiusura della porta d'accesso alla camera ipogea. Come chiusura veniva solitamente impiegato un blocco di tufo o di arenaria dalle dimensioni medie di 1,05 ×0,64 ×0,14 $\mathrm{m}-$ misure queste che si adattano perfettamente a quelle dell'apertura della tomba T.III $(1,00 \times 0,65 \mathrm{~m})-$ ma, più raramente poteva anche essere realizzata mediante la costruzione di un muretto a secco (Benichou-Safar 1982, 
rettangolare d'età bizantina che sancisce il limite orientale del Settore A, è stata solo parzialmente indagata per ragioni di sicurezza. Si tratta certamente di una camera a pianta rettangolare dalle dimensioni ridotte ${ }^{32}$, analogamente alla camera della T.VI descritta di seguito, che, come documentato frequentemente per questo tipo di tombe, ospitava, almeno in origine, una o due sepolture ${ }^{33}$, abitualmente ad inumazione ${ }^{34}$, con i defunti posti direttamente al suolo o entro sarcofagi lignei o, più raramente, di pietra. Dal punto di vista cronologico, questo tipo di tombe è documentato a Cartagine a partire da un periodo compreso tra la metà del VII secolo a.C. e la prima metà del VI secolo a.C. (650-550 a.C. $)^{35}$, ma non può escludersi, data la semplice planimetria, che il tipo non abbia origini più antiche ${ }^{36}$. Prendendo in considerazione la disposizione nello spazio della T.III, perfettamente in asse con le tombe a fossa T.I e T.II, questa è inserita in quello che sembra essere un progetto unitario ${ }^{37}$ del cimitero, con sepolture parallele disposte per file e deve quindi potersi datare ad un momento non molto successivo al VII secolo a.C. e comunque non oltre la prima metà del VI secolo a.C.

Per concludere la descrizione della T.III, è necessario considerare un ultimo elemento che può essere definito anomalo in quanto non documentato per questa tipologia funeraria. Perpendicolare all'entrata F.12 è infatti un secondo pozzetto verticale di forma irregolare approssimativamente rettangolare $(0,8 \mathrm{x} \quad 0,4 \mathrm{~m})^{38}$ profondo $1,05 \mathrm{~m}$ che introduce direttamente alla camera ipogea. Questo sembra essere un accesso secondario, scavato in occasione di un riutilizzo della tomba stessa.

Nella parte più occidentale, è stata identificata e scavata un'ulteriore tomba a camera singola con pozzo d'accesso laterale, la T.VI (fig. 8:2). Differentemente dalle tombe esaminate in precedenza, la tomba in questione presenta un orientamento NE-SO. L'accesso laterale, a sud-ovest, è rettangolare, misura $2,28 \times 1,02 \mathrm{~m}$ ed è profondo $4 \mathrm{~m}$. Lungo tutto il margine nord-occidentale di questo pozzo è un incasso (F.42) ${ }^{39}$ ricavato nel

115, in particolare si veda fig. $61: \mathrm{h})$. Il migliore confronto in questo senso può essere istituito con una tomba a camera con pozzetto d'accesso laterale profondo $2 \mathrm{~m}$ dalla vicina necropoli di Ard-et-Touibi in cui l'ingresso era sigillato mediante un muretto a secco (Poinssot - Lantier 1927, 446-447, tomba n.14). Deve essere inoltre sottolineato che dalla camera della stessa tomba ad Ard-et-Touibi provenivano dei frammenti della cornice che ne adornava l'ingresso: suggestivamente un blocco modanato a dentelli pertinente alla cornice di una tomba punica è stato trovato reimpiegato all'interno dell'edificio d'età bizantina posto sul margine est dell'area di scavo che ha tagliato proprio la camera della tomba T.III.

32 Bénichou-Safar 1982, 117-118. Anche la T.IV deve essere ascritta al medesimo tipo e valgono dunque le riflessioni che verranno proposte di seguito per la tomba T.III.

33 Va comunque notato che le dimensioni esigue della camera non permettono la deposizione primaria di più di due defunti e sono documentate spesso sepolture secondarie con i defunti disposti uno sopra l'altro (si vede solo a titolo d'esempio il caso da Ard-et-Touibi: Poinssot - Lantier 1927, 442, tomba n.3).

34 Sebbene per le tombe di questo tipo l'inumazione sia la modalità di sepoltura più frequente, talvolta è documentato anche il rituale dell'incinerazione o entrambe le pratiche funerarie (Bénichou-Safar 1982, 237).

35 Cintas 1976, 254; Bénichou-Safar 1982, 352.

36 Bénichou-Safar 1982, 352.

37 Cintas 1976, 249.

38 Nella metà orientale i limiti del pozzetto non sono determinabili a causa dei lavori moderni effettuati per il passaggio di una conduttura dell'acqua che ne hanno gravemente danneggiato il perimetro che ora risulta irregolare e frastagliato.

39 Lunghezza 2,28 m, larghezza 0,40 - 0,48 m, altezza 0,05 - 0,07 m. 
piano roccioso che, come per gli aggetti laterali delle tombe a fossa, fu evidentemente scavato per alloggiare e sorreggere le lastre di copertura ${ }^{40}$. Nel pozzo si scendeva attraverso tre pedarole di forma e dimensioni poco regolari ${ }^{41}$ posizionate sulla faccia interna di uno solo dei lati, quello sud-occidentale. Analogamente a quanto riscontrato per la T.III, uno stretto corridoio (F.114) largo $0,72 \mathrm{~m}$, articolato in due bassi gradini conduce, attraverso un'apertura approssimativamente rettangolare (F.98) di $0,72 \times 1,06 \mathrm{~m}$, alla camera ipogea posta ad una quota inferiore. Questa originariamente doveva essere chiusa con una lastra di tufo o arenaria ovvero attraverso un muretto a secco. La camera ipogea (L.120) è a pianta rettangolare e le modeste dimensioni $\left(2,36 \times 1,40 \times 1,44 \mathrm{~m}\right.$; circa 3,3 $\left.\mathrm{m}^{2} ; 4,8 \mathrm{~m}^{3}\right)$ nel caso di sepolture a inumazione non consentono la deposizione primaria di più di due individui ${ }^{42}$. Al suo interno, circa al centro del lato sud-orientale, si trova una piccola nicchia (F.116) di forma irregolare larga $0,40 \mathrm{~m}$ che poteva ospitare la lucerna per il rituale funerario, parte del corredo o, come utilizzo secondario, raccogliere le ossa dei defunti ${ }^{43}$. Nonostante questa tipologia di tombe sia documenta a Cartagine fino alla fine del periodo punico ${ }^{44}$, è difficile ipotizzare per la T.VI un utilizzo primario dopo il V secolo a.C. Anche il diverso orientamento rispetto alla disposizione delle tombe precedentemente descritte, distribuite con criteri spaziali rigorosi, non è un parametro sufficiente per determinare con certezza una variazione nella cronologia in quanto l'orientamento può essere determinato da esigenze quali il naturale declivio ${ }^{45}$. Trovandosi all'interno di un'area funeraria in cui l'insieme delle tombe si data al periodo arcaico, anche la T.VI può essere pertanto ascritta al VI secolo a.C. ${ }^{46}$. Questa interpretazione, d'altra parte, sembra essere supportata dai ritrovamenti all'interno di una cavità irregolare (F.56) scavata nella roccia circa $0,50 \mathrm{~m}$ a nord-est della T.VI e ad essa collegata che ha restituito alcuni reperti ceramici d'età arcaica (fig. 4). Queste ceramiche, spezzate in antico, potrebbero essere la traccia dei rituali funerari che venivano svolti all'esterno della tomba come sembrerebbe documentare la natura stessa dei reperti: una coppa a calotta a velatura rosa a fasce parallele sovradipinte in rosso $^{47}$ ad uso potorio e un mortaio per la preparazione di spezie e alimenti ${ }^{48}$.

La terza e ultima tipologia documentata è rappresentata dalla T.V sita lungo il limite meridionale del Settore A e avente orientamento NNE-SSO. Questa, sebbene debba essere ancora scavata, risulta chiaramente distinguibile dalle altre tombe prese in esame ed è riconducibile al tipo delle tombe costruite a camera singola: "Type X.2:d" della Benichou-

40 Alternativamente potrebbe aver svolto la funzione di piano per la deposizione di una parte del corredo funerario come documentato ad esempio dalla necropoli di Byrsa (Lancel 1999, 75-77, fig. 29).

41 Bénichou-Safar 1982, 121.

42 Sulle pratiche funerarie e le modalità di deposizione dei defunti nelle tombe a camera con accesso laterale si rimanda alle osservazioni fatte per la tomba T.III.

43 Bénichou-Safar 1982, 122.

44 Bénichou-Safar 1982, 352.

45 «There are no homogeneous ritual orientations in the Phoenician Mediterranean, even in the Levant; but each within each settlement the same pattern is followed » (De Jonghe 2016, 107). Sull'orientamento delle tombe nella necropoli di Dermech si veda anche Cintas 1976, 249-250.

46 Sulla cronologia di questo tipo di tombe si rimanda a Cintas 1976, 254; Bénichou-Safar 1982, 352.

47 CA.21.38/1 (fig. 4:1).

48 CA.21.38/2a-b (fig. $4: 2$ ). 
Safar $^{49}$. Documentate a Cartagine non oltre il VI secolo a.C., queste tombe erano erette mediante l'utilizzo di grandi blocchi regolari ben lavorati di arenaria o calcarenite disposti senza malta o leganti. Una sovrastruttura cava a doppia falda imposta sul soffitto della camera realizzata mediante la giustapposizione di blocchi disposti in senso obliquo completava la costruzione. Questa copertura, un elemento strutturale necessario a ridurre la spinta esercitata dal terreno sui muri di sostegno, non era a vista ed era solitamente chiusa da un alto muro di facciata costruito a file di blocchi sovrapposti. Della T.V, parzialmente obliterata da una serie di costruzioni d'epoca successiva (M.5, L.72, M.53), sono ancora visibili 4 dei grandi blocchi giustapposti $(0,7 \times 0,4 \times 0,2 \mathrm{~m})$ che costituivano la copertura a doppio spiovente e un blocco perpendicolare $(0,4 \times 0,3 \times 0,3 \mathrm{~m})$ disposto di taglio che doveva essere parte del muro di chiusura (fig. 3).

\subsection{Settore A: Stratigrafia e ritrovamenti}

Come accennato all'inizio del precedente paragrafo, le tombe del Settore A sono state trovate prive dei loro contesti originali, ma tra di esse possono essere operate delle distinzioni e avanzate delle ipotesi interpretative. Nelle tombe T.I, T.II, T.III e T.VI gli strati di riempimento sono molto simili tra loro in quanto caratterizzati da terra marrone scura rimescolata con materiali misti. È evidente che queste tombe, dopo lo scavo degli anni ' 50 , siano state colmate dalla terra dilavata degli strati superiori circostanti. Emblematici in questo senso sono i ritrovamenti all'interno del pozzo d'accesso laterale (US.50) e dentro la camera ipogea L.120 (US.112) della T.VI che ha restituito il maggior numero di materiali (figg. 9-10). Si tratta di reperti attribuibili ai periodi tardo imperiale e bizantino (III-VIII secolo d.C.) - con alcuni elementi residuali d'età tardo punica (III-II secolo a.C.) - tra i quali figurano principalmente ceramiche di produzione africana, ma anche di Pantelleria, accompagnate da oggetti di bronzo, di ferro e piccoli contenitori di vetro insieme ad elementi architettonici quali tubuli e porzioni frammentarie degli intonaci e delle decorazioni musive degli edifici limitrofi.

Il riempimento della T.VIII (US.54, US.106), al contrario, era uno strato sabbioso di colore giallo con lenti di colore rosso-marrone che presentava al suo interno materiali ben databili al periodo tardo-punico (III-II secolo a.C.) (fig. 11). Sembra dunque evidente che questa tomba sia stata violata in antico dopo la distruzione di Cartagine del 146 a.C. probabilmente al momento dell'impianto delle strutture d'età romana - e che non sia stata scavata nel corso delle indagini condotte da M. Vezat: la T.VIII può dunque fornire un indizio di come le tombe furono trovate dall'archeologo francese ${ }^{50}$. Tra i materiali della T.VIII, inoltre, sono stati recuperati due vasi che potrebbero essere ricondotti al corredo funerario originale: si tratta di un piatto fenicio Red Slip di produzione locale ad ampia tesa inclinata (fig. 11:2) ${ }^{51}$, un tipo ben documentato tra la fine dell'VIII secolo a.C. e la metà del successivo, e una coppa carenata realizzata e brunita a mano con ingubbiatura rossa estesa a

\footnotetext{
Bénichou-Safar 1982, 144 e 153-162. Sul tipo si veda anche Cintas 1976, 271-277, figg. 25-26.

Situazione analoga è ipotizzabile per la T.IX.

51 CA.21.54/1.
} 
tutta la superficie dal riempimento (US.106) della nicchia F.96 che può ben datarsi nello stesso intervallo cronologico (fig. 11:1) ${ }^{52}$.

Procedendo nella disamina delle evidenze archeologiche del Settore A, vengono presi in esame a seguire i resti che non sono strettamente correlati alle sepolture d'epoca fenicia. Costruiti direttamente sopra la roccia vergine che ospita la necropoli di Dermech, in diversi punti del Settore A, sono stati individuati residui di pavimentazioni in cocciopesto di ottima fattura (L.28, L.48, L.72). È difficile stabilire se questi fossero in fase tra loro o quantomeno coevi, specialmente in considerazione del fatto che i materiali in giacitura primaria relativi a questi piani pavimentali, se non sono stati dispersi nel corso dei secoli, sono certamente stati ritrovati durante gli scavi degli anni ' 50 . Al momento solamente L.72 e il soprastante strato di crollo US.70 consentono di avere informazioni attendibili. Il piano, situato nella porzione centro meridionale del Settore $\mathrm{A}$, è conservato in prossimità dell'angolo di una struttura muraria completamente rasata (M.51; M.53). Questa è stata costruita a ridosso della T.V della quale segue l'orientamento NNE-SSO (fig. 3). Il limitato stato di conservazione ha consentito il ritrovamento di un numero esiguo di reperti diagnostici; tuttavia, la presenza di un unguentario piriforme ${ }^{53}$ e di una kakkabe $e^{54}$ consentono di datare lo strato con una certa precisione tra il III e il II secolo a.C.

Nella porzione orientale del Settore A, al di sopra delle tombe T.III e T.IV, è un piano battuto intonacato di bianco (L.36). Questo si lega ad est al muro M.37 che risulta tagliato perpendicolarmente a nord da una grande struttura (M.31) realizzata con pietre di piccole dimensioni disposte irregolarmente (opus incertum). In questo piccolo angolo la stratigrafia è intatta e sono stati ritrovati in crollo (US.34) una serie di vasi interi o parzialmente ricostruibili allettati sul pavimento L.36 che si datano puntualmente tra la metà del III e la prima metà del II secolo a.C. (250-146 a.C.) (fig. 12) $)^{55}$.

Una situazione stratigrafica differente e più articolata è invece riscontrabile nella sezione sud-occidentale del Settore A (figg. 5-6). In seguito all'ampliamento del limite di scavo verso sud, infatti, è stato possibile rintracciare una sequenza stratigrafica completa, non inficiata dagli interventi di epoca romana e bizantina, né dagli scavi degli anni ' 50 . Dal momento che quest'area non è stata indagata in modo estensivo i dati forniti di seguito devono essere considerati preliminari. Sotto lo strato di humus moderno profondo pochi centimetri, insiste immediatamente un esteso strato di crollo (US.74) compatto e di colore rosso $^{56}$. Questo poggia sopra un piano battuto denominato L.76 compreso tra due muri paralleli (M.47, M.49) con andamento NE-SO. A giudicare dai rinvenimenti (fig. 13), in corrispondenza dei due muri è possibile vi fosse una porta: sotto ad una pietra in crollo a

52 CA.21.106/1

53 CA.21.70/2. Sugli unguentari piriformi con base a bottone si vedano come confronto esemplari analoghi ritrovati nella stessa Cartagine per i quali si rimanda a: Vegas 1999, 172-173, fig. 75:4-5; Bechtold 2007, 372 , fig. 187:2178

54 CA.21.70/3. Si vedano come confronto gli esemplari analoghi ritrovati a Cartagine per i quali si rimanda a: Vegas 1999, 197, fig.106:3; Bechtold 2007, 421, fig. 220:2366

$55 \mathrm{Si}$ tratta principalmente di tre unguentari piriformi con piede a bottone e decorazione lineare dipinta in rosso sulla spalla (CA.21.34/1, /2, /3), una kakkabe (CA.21.34/4) e una coppa a vernice nera outturned rim (CA.21.34/5)

56 Lo strato US.74 per colore, consistenza e composizione deve essere avvicinato allo strato di crollo US.70. 
ridosso di M.49 erano, infatti, un cardine ${ }^{57}$, una chiave o raccordo ${ }^{58}$ e cinque rivetti di bronzo $^{59}$. Al momento, l'unico elemento che accompagnava questi oggetti di bronzo è il coperchio di un'anfora e la prima metà del II secolo a.C. è il periodo più attendibile entro cui datare i reperti presenti nello strato di crollo. Sotto la pavimentazione L.76, si susseguono una serie di strati (US.78, US.86) databili tra il IV e il III secolo a.C. Particolarmente rilevanti al fine di una possibile interpretazione cronologica sono una calyx-cup a vernice nera frammentaria con decorazione plastica raffigurante un volto grottesco di probabile produzione campana dalla US.78 (350-300 a.C.) ${ }^{60}$ (fig. 14:1) e, dalla US.86, due coppe a vernice nera outturned rim di produzione locale databili tra il 350 e il 250 a.C. ${ }^{61}$. Appena sotto l'US.86, due strutture rasate in mattone crudo (M.55 ed M.57) si trovano esattamente in corrispondenza dei muri M.47 e M.49 e le successive unità stratigrafiche si dispongono rispettivamente a est e ad ovest di M.55. Sul lato est, sono stati individuati due strati (US.92, in fase con la canaletta B.63 realizzata con le pareti di almeno due diverse anfore puniche a siluro e US.102) databili sulla base dei materiali ceramici tra la metà del V e gli inizi del IV secolo a.C. (fig. 14:2-8): tra le forme diagnostiche sono documentate una large stemless cup attica a vernice nera con caratteristico motivo a palmette stampigliate sul fondo (450-425 a.C. $)^{62}$, due piattini ombelicati di tradizione punica con bande concentriche dipinte di rosso sulla tesa in prossimità dell'orlo ${ }^{63}$, una cythra ${ }^{64}$ e alcune anfore puniche afferenti ai tipi T-4.1.1.3 ${ }^{65}$ e T-1.4.4.1 ${ }^{66}$. Nel lato ovest, invece, si delinea una situazione differente. Gli strati US.94 e US.104, oltre a presentare materiali più antichi che si datano tra la seconda metà del VI e la prima metà del V secolo a.C. ${ }^{67}$, sono connotati dall'elevata quantità di scarti di lavorazione e di contatori verosimilmente ricollegabili ad un'istallazione industriale nell'area (fig. 15).

\subsection{Settore B: il saggio di scavo}

Alle spalle del Settore A, a circa $10 \mathrm{~m}$ di distanza procedendo verso sud, è stato aperto un saggio di scavo di $5 \times 5 \mathrm{~m}$ per indagare l'estensione della necropoli e studiare la stratigrafia in un punto in cui la roccia vergine si approfondisce. Sotto il sottile

57 CA.21.86. Sui reperti di bronzo da Cartagine si rimanda a Mansel 2007, 796-813.

58 CA.21.87. Si veda per confronto Mansel 2007, 810, fig. 448:6509, 6512.

59 CA.21.53; CA.21.56; CA.21.85; CA.21.88; CA.21.89. Si veda per confronto Mansel 2007, 810, fig. 448:6509.

60 CA.21.78/1. Per un confronto si veda Sparkes - Talkot - Richter 1970, 121-122, 285, nn. 694-695 e tav. 28:694-695.

61 CA. $21.86 / 2$, CA.21.86/5.

62 CA.21.92/1 (fig. 14:2). Motivo decorativo costituito da una serie palmette a 12 petali collegate alla base da festoni che racchiudono un motivo radiale sul fondo della vasca. Il confronto puntuale è in Sparkes - Talkot Richter $1970,104,269$, n. 486 e tav. 50:486.

63 CA.21.92/3; CA.21.102/1 (fig. 14:3, 6). Per alcuni possibili confronti si rimanda a Vegas 1999, 138, fig. 26:1$2,4$.

64 CA.21.102/5 (fig. 14:7). Per un confronto si veda Vegas 1999, 192, fig. 9:4.

65 CA.21.92/6a-b (fig. 14:4). Ramon Torres 1995, 185-186, T-4-1.1.3, fig. 38

66 CA.21.92/7; CA.21.102/11 (fig. 14:5, 8). Ramon Torres 1995, 175-176, T-1-4.4.1, fig. 18.

67 Tra questi si annoverano una coppa ionica tipo B2 frammentaria (CA.21.104/6; fig. 15:13), un piatto ombelicato a tesa ampia con fascia dipinta di rosso sull'orlo (CA.21.94/2; fig. 15:10), l'orlo di una oil bottle fenicia (CA.21.94/6, fig. 15:9) e una serie di olle a velatura gialla o rosa attribuibili al periodo tardo arcaico (CA.21.104/1, /2, /13, fig. 15:14). 
riempimento moderno privo di materiali diagnostici, sono distinguibili due strati (US.302, 304). Quello più superficiale, la US.302, caratterizzato da terra marrone-scuro friabile, è ricco di materiali rimescolati ascrivibili ad epoche diverse. La sottostante US.304, di colore marrone-rossastro, che era interessata da un fitto intreccio di radici, ha restituito materiali ascrivibili ai secoli III-V d.C. Dopo l'asportazione di questa unità stratigrafica, raggiunta su tutta l'area quota $-0,60 \mathrm{~m}$ rispetto al piano di calpestio moderno, non sono ancora distinguibili strutture o pavimentazioni ad eccezione di un unico grande blocco spezzato d'arenaria di forma triangolare (M.305) posto nel settore nord-orientale del saggio di scavo.

\section{CONCLUSIONI}

I nuovi dati ottenuti durante la prima campagna di scavi a Cartagine della Missione congiunta dell'Institut National du Patrimoine e dell'Università degli Studi di Roma «La Sapienza», sebbene preliminari, consentono di riflettere con maggiore chiarezza sullo sviluppo della necropoli di Dermech in questo punto del parco delle Terme di Antonino. L'orientamento E-O della maggior parte delle tombe, sia a fossa sia a camera (T.I, II, III, IV, VII), mostra come, a partire da un momento avanzato del VII secolo a.C., a Dermech fosse già avviato un piano architettonico unitario del cimitero, mentre l'attribuzione delle tombe a tipologie esatte permette di riflettere sui cambiamenti sociali e culturali che hanno interessato Cartagine durante il periodo arcaico (VII-VI secolo a.C.) e sui rituali funerari adottati dalla comunità fenicia in quest'area. I materiali provenienti dalla sezione meridionale del Settore A, inoltre, forniscono nuove informazioni sull'occupazione del settore di Dermech nel corso dei secoli successivi all'utilizzo della necropoli e consentono di ipotizzare la presenza di istallazioni industriali a partire almeno dal V secolo a.C.

\section{BIBLIOGRAFIA}

AleXANDER, M.A. - Ben Abed-Ben KHAdER, A. - GuY, P.R.

1996 The corpus of the Mosaics of Tunisia: Carthage Project 1992-1994: Dumbarton Oaks Papers 50 (1996), pp. 361-368.

AOUNALLAH, S.

2018 La fondation de la Colonia Concordia Iulia Carthago et l'aménagement de la colline de Byrsa: S. Aounallah - A. Mastino (éd.), Carthage : Maîtresse de la Méditerranée, Capitale de l'Afrique (IX siècle avant J.-C. - XIIe siècle) (Histoires et Monuments 1), Tunis 2018, pp. 238-243.

Aounallah, S. - BaKLOUTI, H. - Mosca, A.

2018 Le complexe hydraulique Zaghouan-Carthage: S. AounALlaH - A. MASTINO (éd.), Carthage : Maîtresse de la Méditerranée, Capitale de l'Afrique (IX siècle avant J.-C. - XIIe siècle) (Histoires et Monuments 1), Tunis 2018, pp. 278-287.

BARATTE, F.- BEJAOUI, F.

2018 Carthage Byzantine: S. AounAllah - A. MAStino (éd.), Carthage : Maitresse de la Méditerranée, Capitale de l'Afrique (IX siècle avant J.-C. - XIIe siècle) (Histoires et Monuments BARTOLONI, P. 1), Tunis 2018, pp. 371-387.

1973 Necropoli puniche della costa nord-orientale del Capo Bon: E. ACQUARO - P. BARTOLONI - A. Ciasca - M.H. FAnTAR (edd.), Prospezione archeologica al Capo Bon I (Collezione di Studi Fenici 2), Roma 1973, pp. 9-68. 
1985 Monte Sirai 1984. La necropoli (campagne 1983 e 1984): Rivista di Studi Fenici XIII/2 (1985), pp. 247-263.

2000 La necropoli di Monte Sirai - I (Collezione di Studi Fenici 41), Roma 2000.

BECHTOLD, B.

2007 Die phönizisch-punische Gebrauchskeramik der archaischen bis spätpunischen Zeit: H.G. NIEMEYER - R.F. DOCTER - K. SCHMIDT - B. BECHTOLD (hrsg.), Karthago. Die Ergebnisse der Hamburger Grabung unter dem Decumanus Maximus (Hamburger Forschungen zur Archäologie, 2), Mainz am Rhein 2007, pp. 327-431.

BEN ABED-BEN KHADER, A.

1999 Karthago. Les mosaïques du parc archéologique des Thermes d'Antonin (Corpus des Mosaïques de Tunisie vol. IV, 1), Tunis 1999.

BENICHOU-SAFAR, $\mathrm{H}$.

1982 Les tombes puniques de Carthage. Topographie, structures, inscriptions et rites funéraires Вотто, M. (Études d'Antiquités africaines 3), Paris 1982.

2008 Forme di interazione e contatti culturali fra Cartagine e la Sardegna sudoccidentale nell'ambito del mondo funerario: J. GONZALEZ - P. RUGGERI - C. VISMARA - R. ZuCCA (edd.), L'Africa Romana. Atti del XVII Convegno Internazionale di Studi: Le ricchezze dell'Africa, Sevilla, 14-17 dicembre 2006 (Collana del Dipartimento di storia dell’Università degli studi di Sassari, Nuova serie 35), Roma 2008, pp. 1619-1631.

CADOTTE, A.

2007 La Romanisation des Dieux. L'interpretatio romana en Afrique du Nord sous le Haut-Empire (Religions in the Graeco-Roman world, vol. 158), Leiden - Boston 2007.

CINTAS, P.

1970 Manuel d'Archéologie Punique I. Histoire et archéologie comparées. Chronologie des temps archä̈ques de Carthage et des villes phéniciennes de l'ouest (Collection des manuels d'Archéologie et d'Historie de l'Art), Paris 1970.

1976 Manuel d'Archéologie Punique II. La civilisation carthaginoise. Les réalisations matérielles (Collection des manuels d'Archéologie et d'Historie de l'Art), Paris 1976.

DAUX, A.

1869 Recherches sur l'origine et l'emplacement des emporia Phéniciens dans le Zeugis et le byzacium, Paris 1869

DE JONGHE, $\mathrm{M}$.

2016 Phoenician Architecture and Funeral Customs in North Africa. A Comparison of Utica and Carthage: H. TÖPFER - F. SCHÖN (hrsg.), Karthago Dialoge. Karthago und der punische Mittelmeerraum - Kulturkontakte und Kulturtransfers im 1. Jahrtausend vor Christus (RessourcenKulturen Band 2), Tübingen 2016, pp. 101-118.

DELATTRE, R.P.

1896 Carthage. Nécropole Punique de la Colline de Saint-Louis, Lyon 1896.

FANTAR, M.

2002 Recherches sur l'architecture funéraire punique du Cap Bon (Collezione di Studi Fenici 42), Roma 2002.

FARISELLI, A.C.

2006 Il «paesaggio» funerario: tipologie tombali e rituali funerari a Tharros: E. ACQUARO - C. DEL VAIS - A.C. FARISELLI (edd.), La necropoli meridionale di Tharros. Tharrica I (Biblioteca di Byrsa 4), Sarzana 2006, pp. 303-369.

GAUCKLER,P.

$1915 \quad$ Nécropoles Puniques de Carthage, Vol. II, Paris 1915. 
GuIRGUIS, $\mathrm{M}$.

2010 Necropoli fenicia e punica di Monte Sirai. Indagini archeologiche 2005-2007 (Studi di Storia KLEIJWEGT, M Antica e di Archeologia 7), Ortacesus 2010.

1994 Beans, baths and the barber...a sacred law from Thuburbo Maius: Antiquités africaines 30 (1994), pp. 209-220.

LANCEL, $\mathrm{S}$.

1989 Victor de vita et la Carthage vandale: A. MASTINO (ed.), L'Africa Romana: Atti del VI convegno di studio (Sassari, 16-18 dicembre 1988) (Collana del Dipartimento di storia dell'Università degli studi di Sassari, Nuova serie 14), Sassari 1989, pp. 649-661.

$1999 \quad$ Carthage (Africana), Tunis 1999.

MANSEL, K.

2007 Metallfunde: H.G. NIEMEYER - R.F. DOCTER - K. SCHMIDT (hrsg.), Karthago. Die Ergebnisse der Hamburger Grabung unter dem Decumanus Maximus II (Hamburger Forschungen zur Archäologie, 2), Mainz am Rhein 2007, pp. 796-813.

Maraoui-Telmini, B. - ChElbi, F. - Docter, R.F

2013 Les fouilles tuniso-belges du terrain Bir Massaouda (2002-2005) : contribution à la connaissance de la topographie de Carthage à l'époque archaïque: A.M. ArRudA (éd.), Fenícios e Púnicos, Por Terra e Mar, Actas do VI Congresso Internacional de Estudos Fenícios e Púnicos, (Lisboa 25 de Setembro a 1 de Outubro de 2005), vol. 2, Lisboa 2013, pp. 906-916.

PICARD, G.-CH

1949 Rapport sur l'archéologie romaine en Tunisie Durant le premier semestre 1948: Bulletin archéologique du Comité des travaux historiques et scientifiques 1949, pp. 506-529.

1952 Rapport sur l'archéologie romaine en Tunisie Durant le premier semestre 1950: Bulletin archéologique du Comité des travaux historiques et scientifiques 1952, pp. 152-161.

POINSSOT, L. - LANTIER, R.

1927 Fouilles à Carthage: Bulletin archéologique 1927, pp. 437-474.

RAMON-TORRES, J.

1995 Las ánforas fencio-púnicas del Mediterráneo central y occidental, Barcelona 1995.

SECCI, R.

2016 Nuovi tipi tombali nella necropoli meridionale di Tharros (campagna di scavo 2015): Byrsa. Scritti sull'antico Oriente mediterraneo 25-26, $27-28$ (2016), pp. 185-202.

SPARKES, B.A. - TALKOT, L. - RICHTER, G.M.A.

1970 Black and plain pottery of the $6^{\text {th }}, 5^{\text {th }}$ and $4^{\text {th }}$ centuries BC (The Athenian Agorà XII), Princeton 1970.

VEGAS, M.

1999 Phöniko-punische Keramik aus Karthago: F. RAKOB (hrsg.), Karthago III. Die Deutschen VATTONI, F. Ausgrabungen in Karhtago, Mainz am Rhein 1999, pp. 93-219.

1987 Appunti africani: Studi Magrebini 10 (1978), pp. 10-31.

VERNAZ, J.

1887 Note sur des fouilles à Carthage 1884-1885: Revue Archéologique 10 (1887), pp. 151-170.

VEZAT, M.

1969 Fouilles inédites. Comment Carthage enterrait ses morts aux VIè et Vè siècles: Archéologie Vivante I/2 (1969), pp. 66-72, p. 154. 


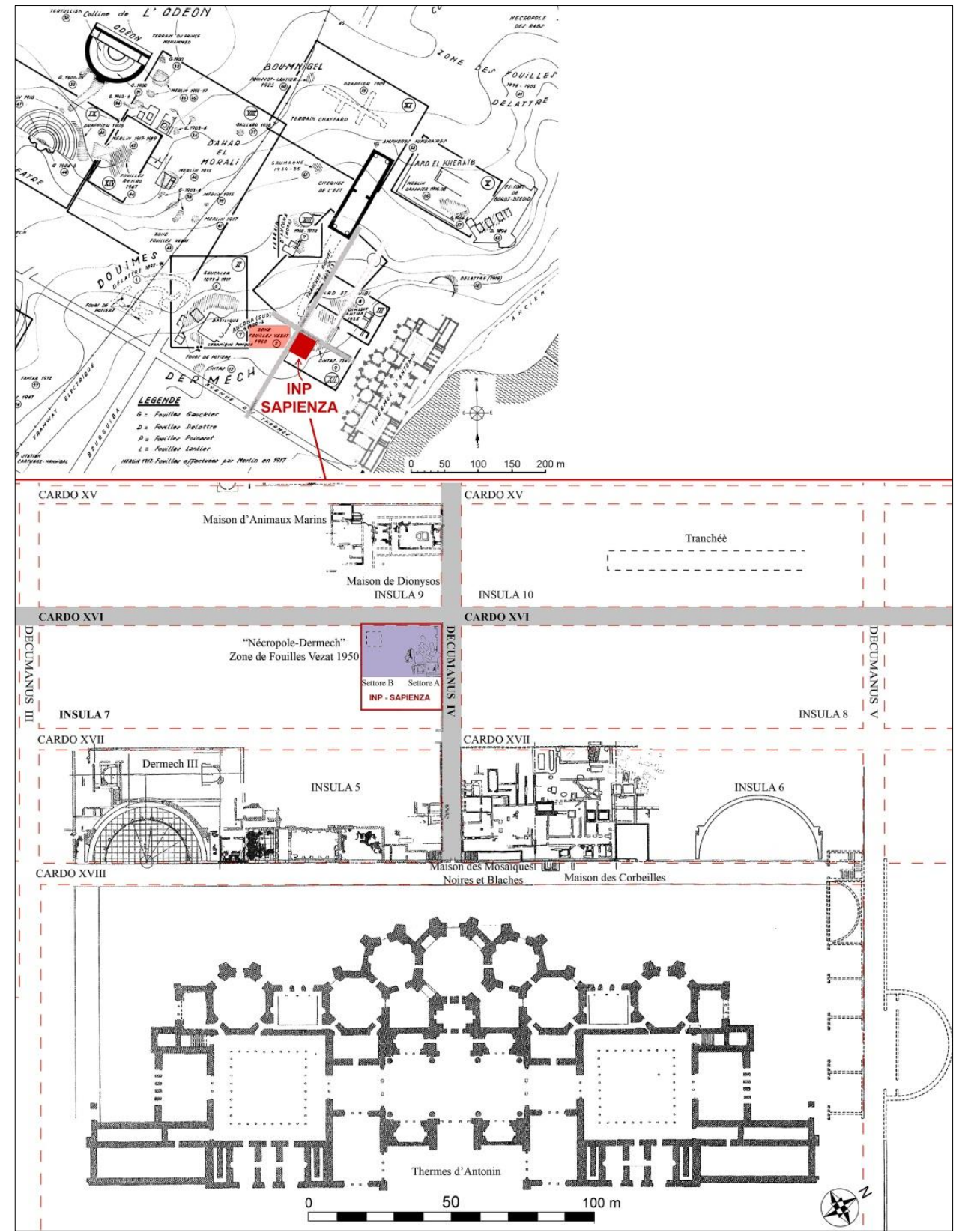

Fig. 1 - Dettagli della pianta di Cartagine (da Benichou-Safar 1982, fig. 3 e Ben Abed-Ben Khader 1999, pianta 1) con evidenziata l'area della necropoli di Dermech interessata dalla prima campagna di scavi (2021) della Missione a Cartagine dell'INP - Sapienza. 


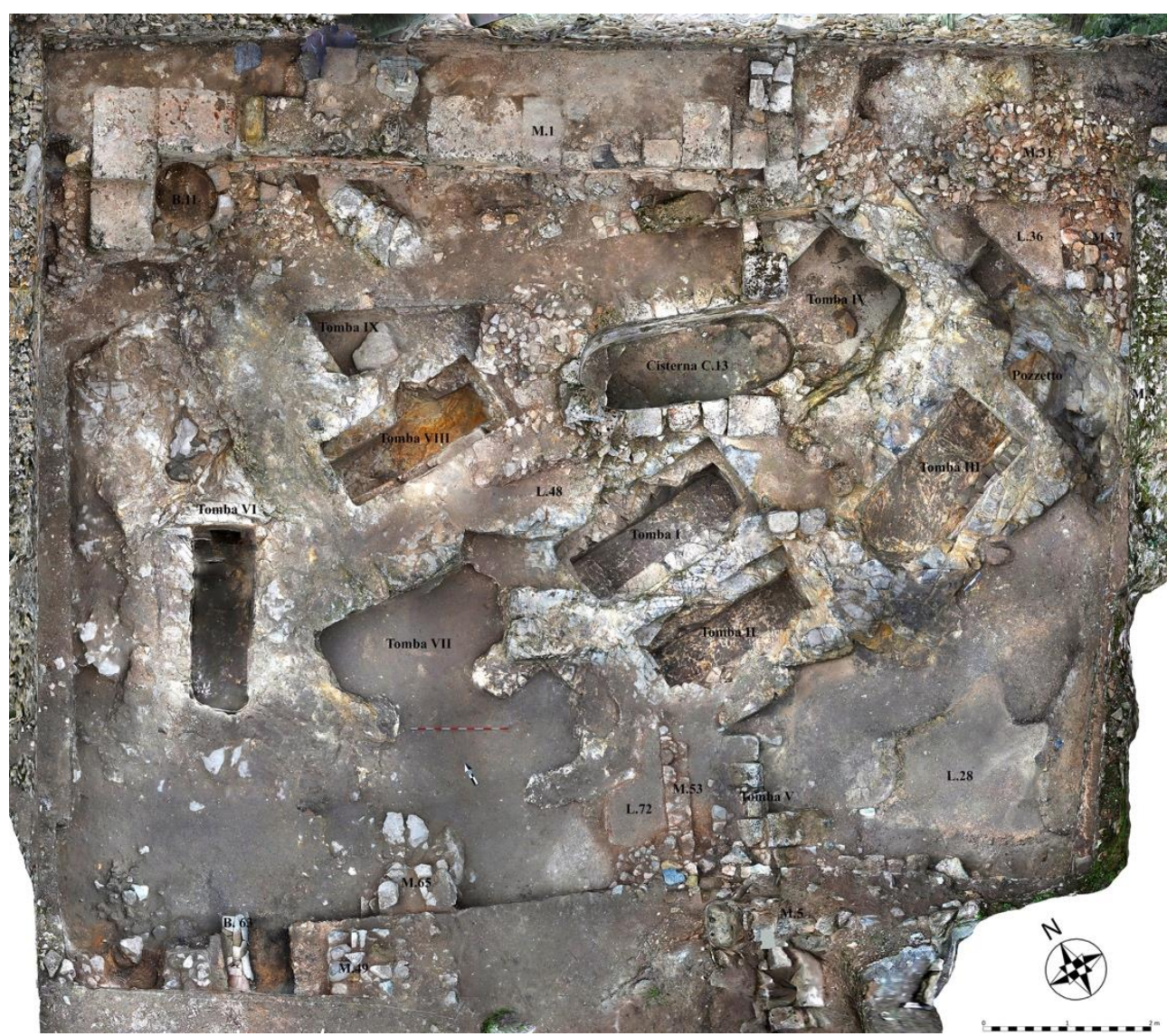

Fig. 2 - Nècropole - Dermech, ortofoto del Settore A. Al centro le tombe dopo lo scavo. 


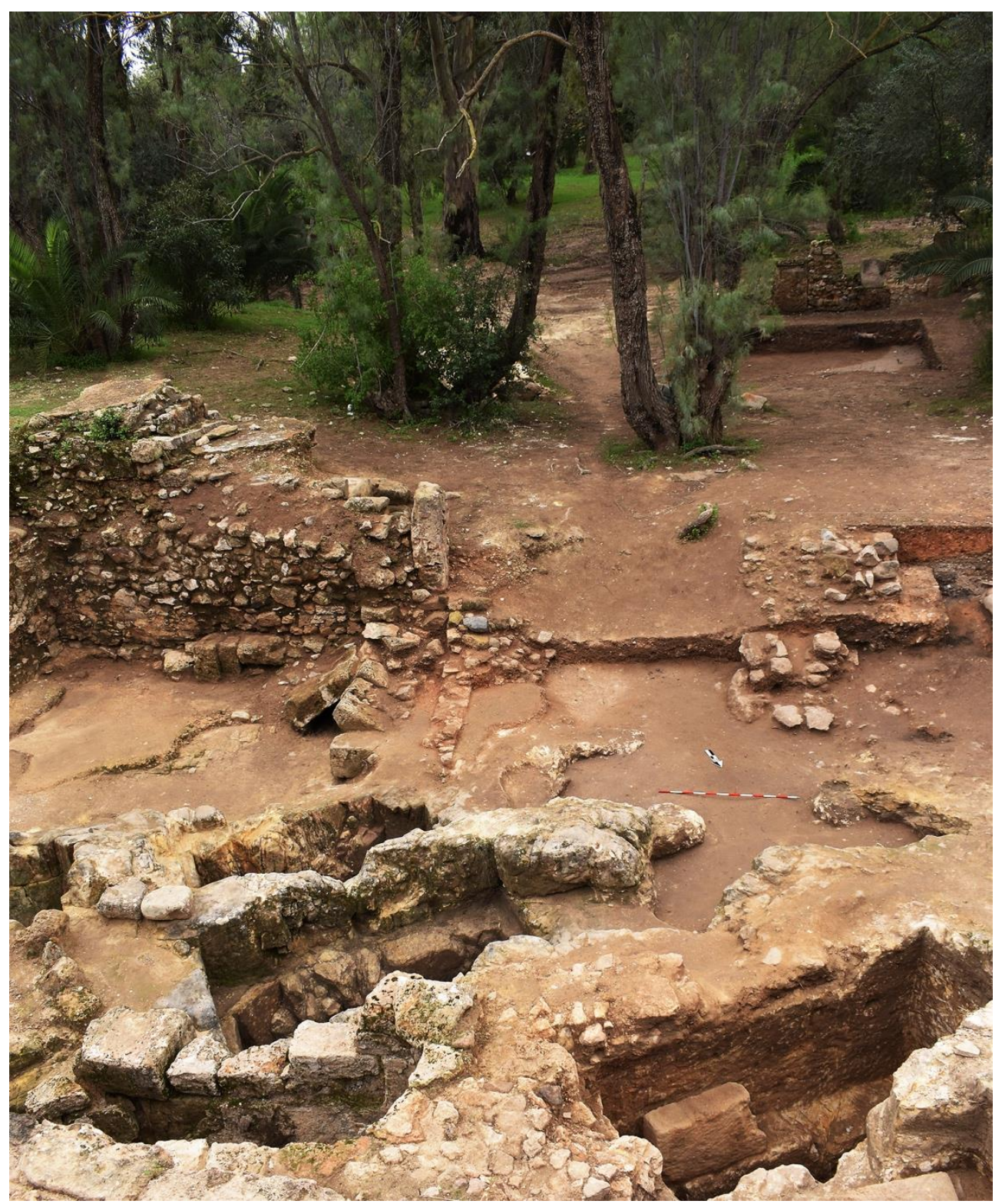

Fig. 3 - Nècropole - Dermech, Settore A (vista da nord-est): in primo piano la T.VIII con la lastra M.45 (sulla destra), e le T. I e T.II (a sinistra). Al centro a sinistra la tomba costruita T.V con copertura a doppio spiovente. Sullo sfondo il saggio di scavo nel Settore B. 

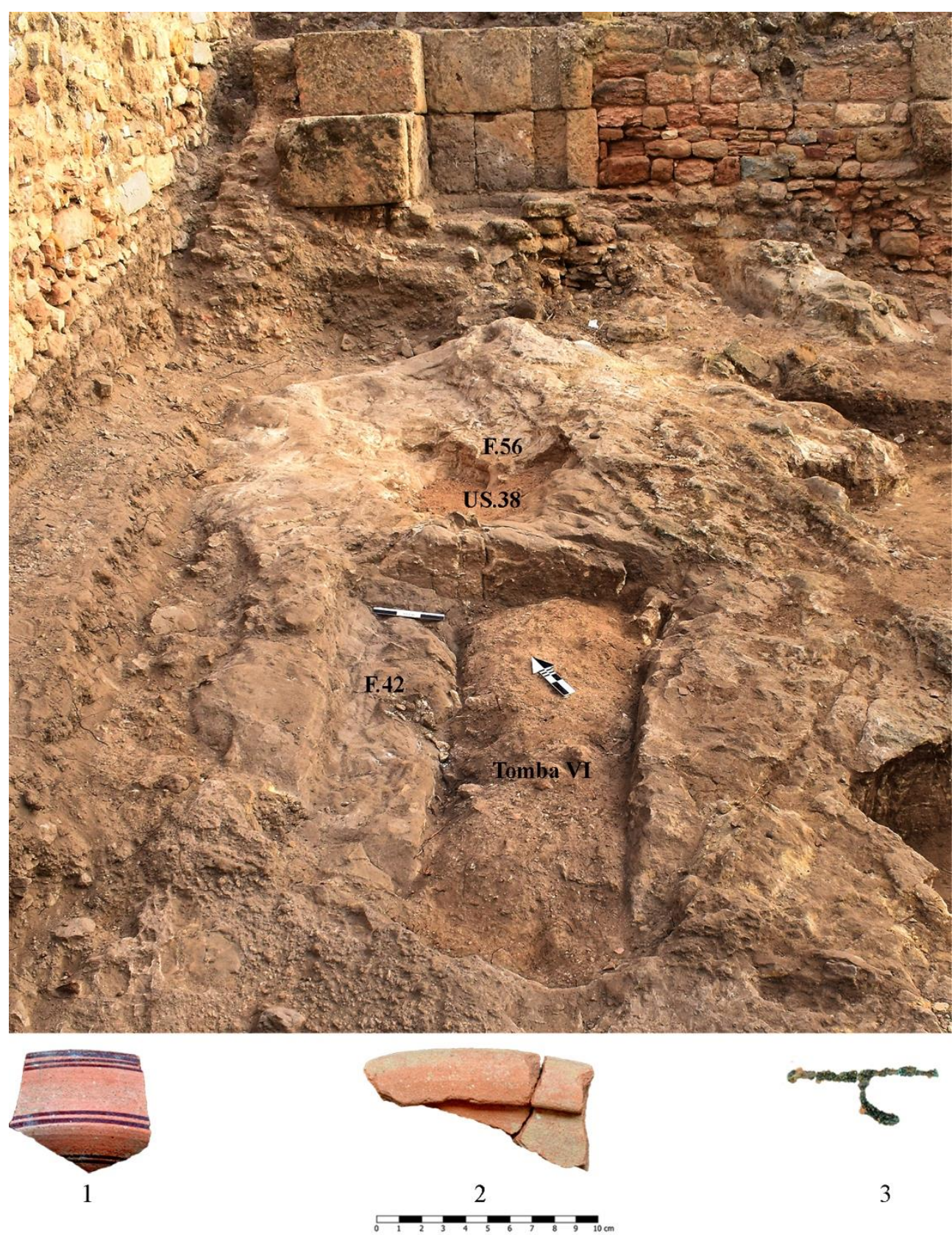

3

Fig. 4 - Nècropole - Dermech, Settore A: dettaglio della T.VI e dell'adiacente cavità F.56 in corso di scavo (vista da sud-ovest). In basso i reperti provenienti dalla US.38. 


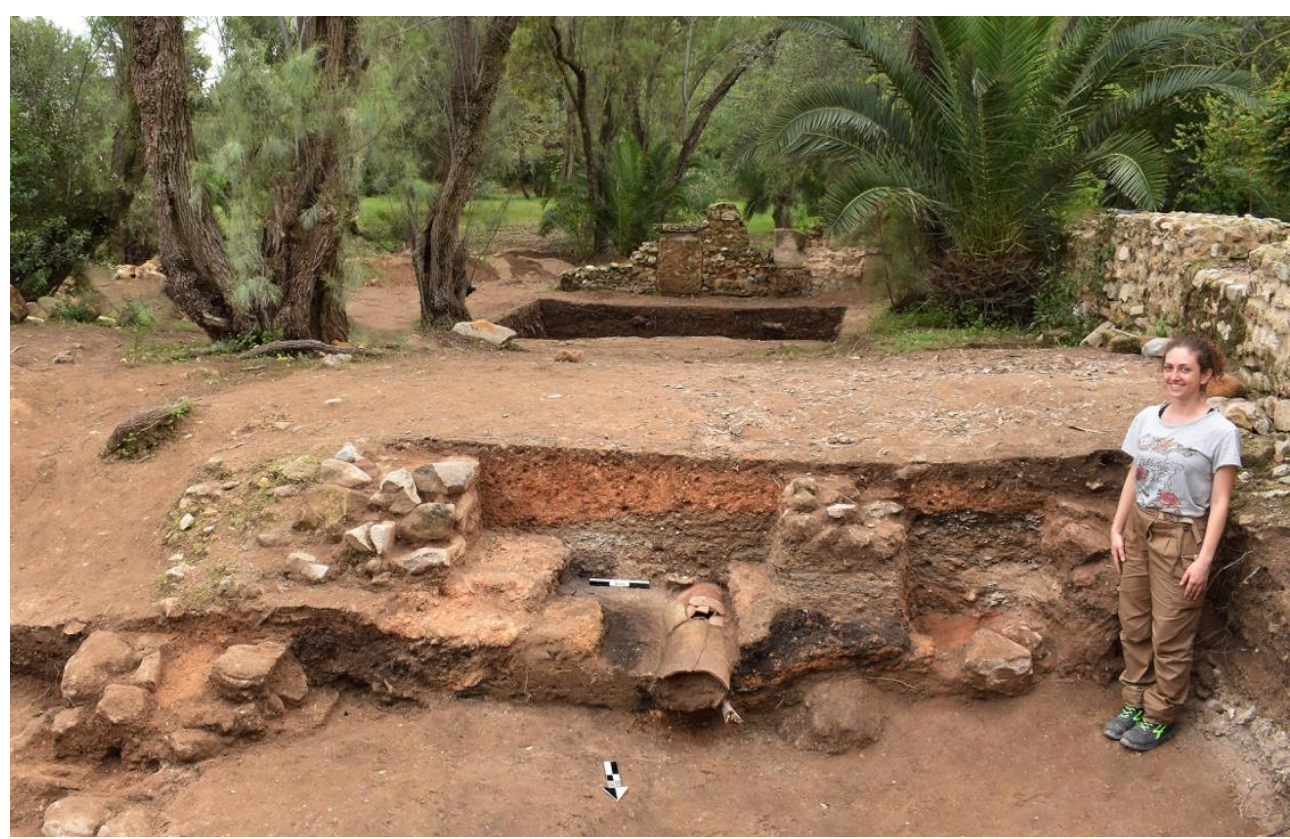

Fig. 5 - Nècropole - Dermech, Settore A: sezione meridionale del saggio di scavo nella metà occidentale (vista da nord-est).

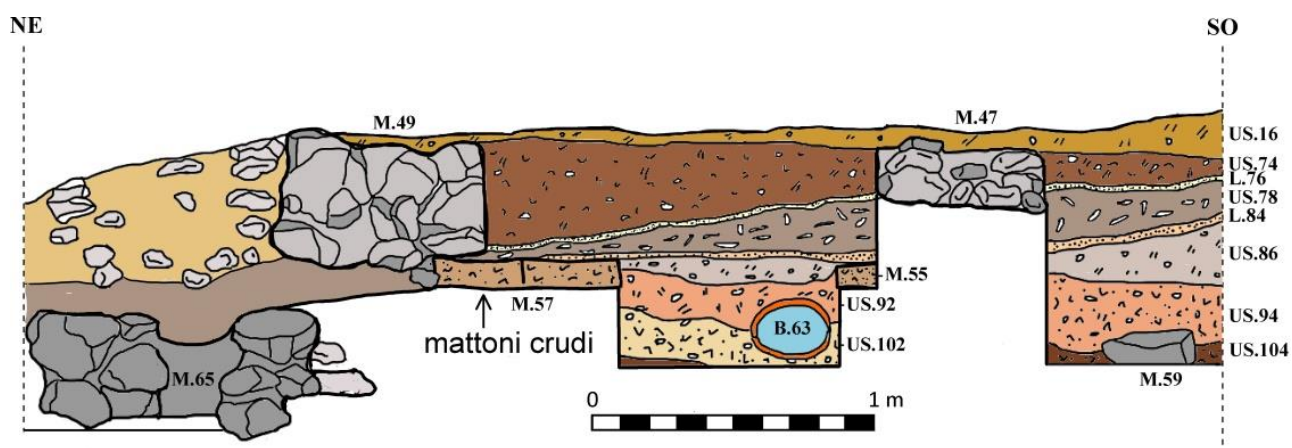

Fig. 6 - Nècropole - Dermech, Settore A: sezione stratigrafica meridionale del saggio di scavo nella metà occidentale. 

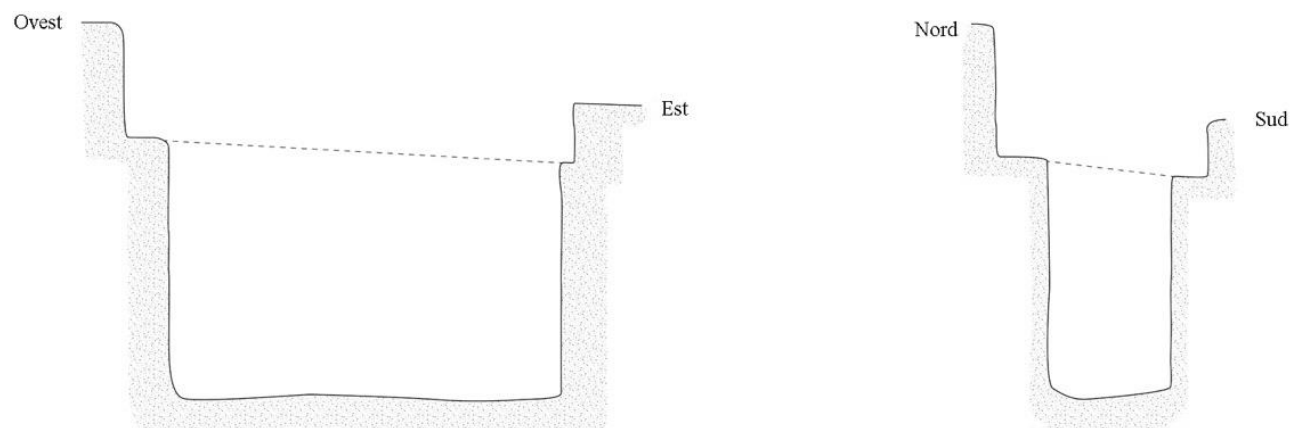

1. Tomba T.I
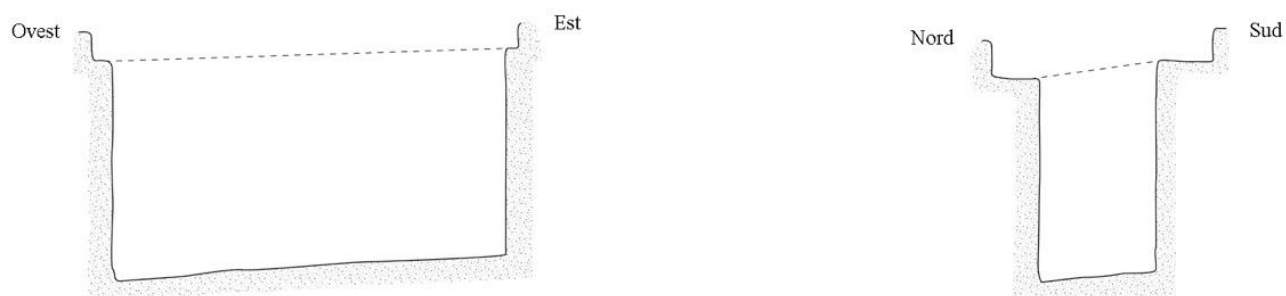

2. Tomba T.II

Ovest
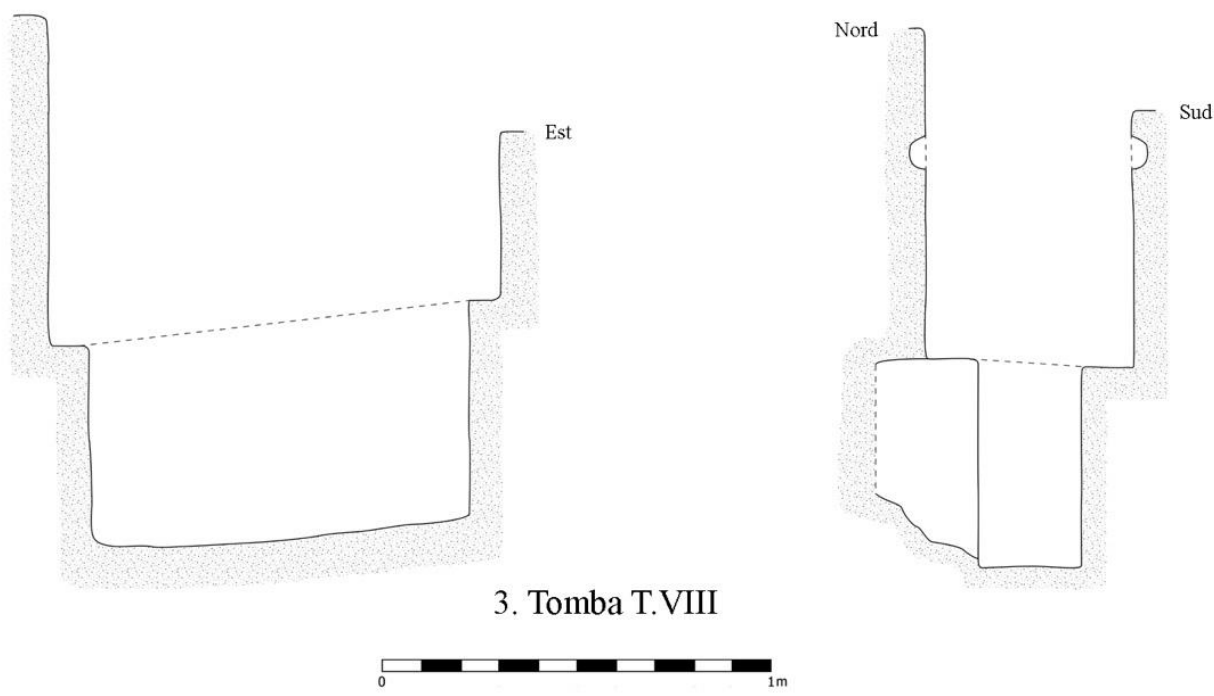

Fig. 7 - Nècropole - Dermech, Settore A: tombe a fossa con aggetti laterali, sezioni (scala 1:20). 

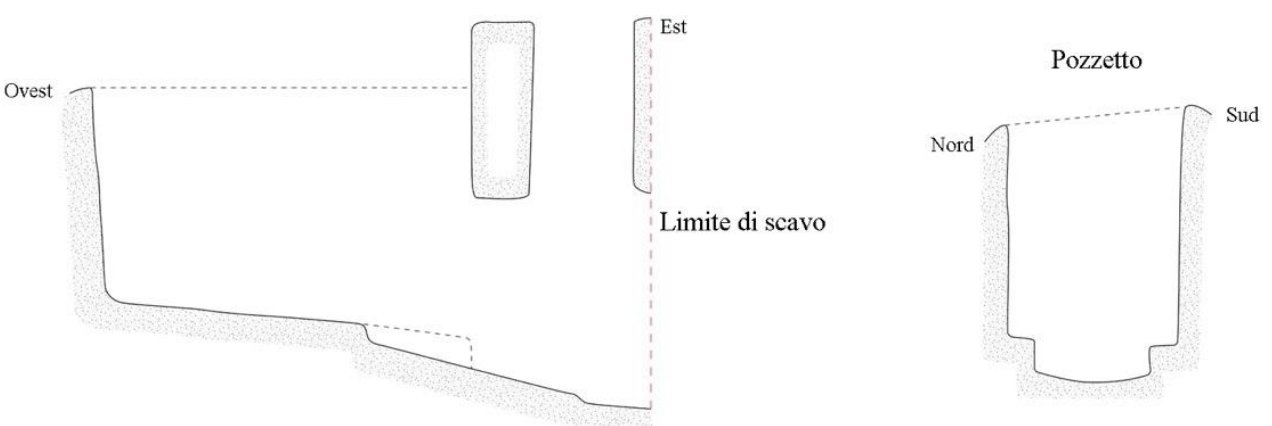

1. Tomba T.III

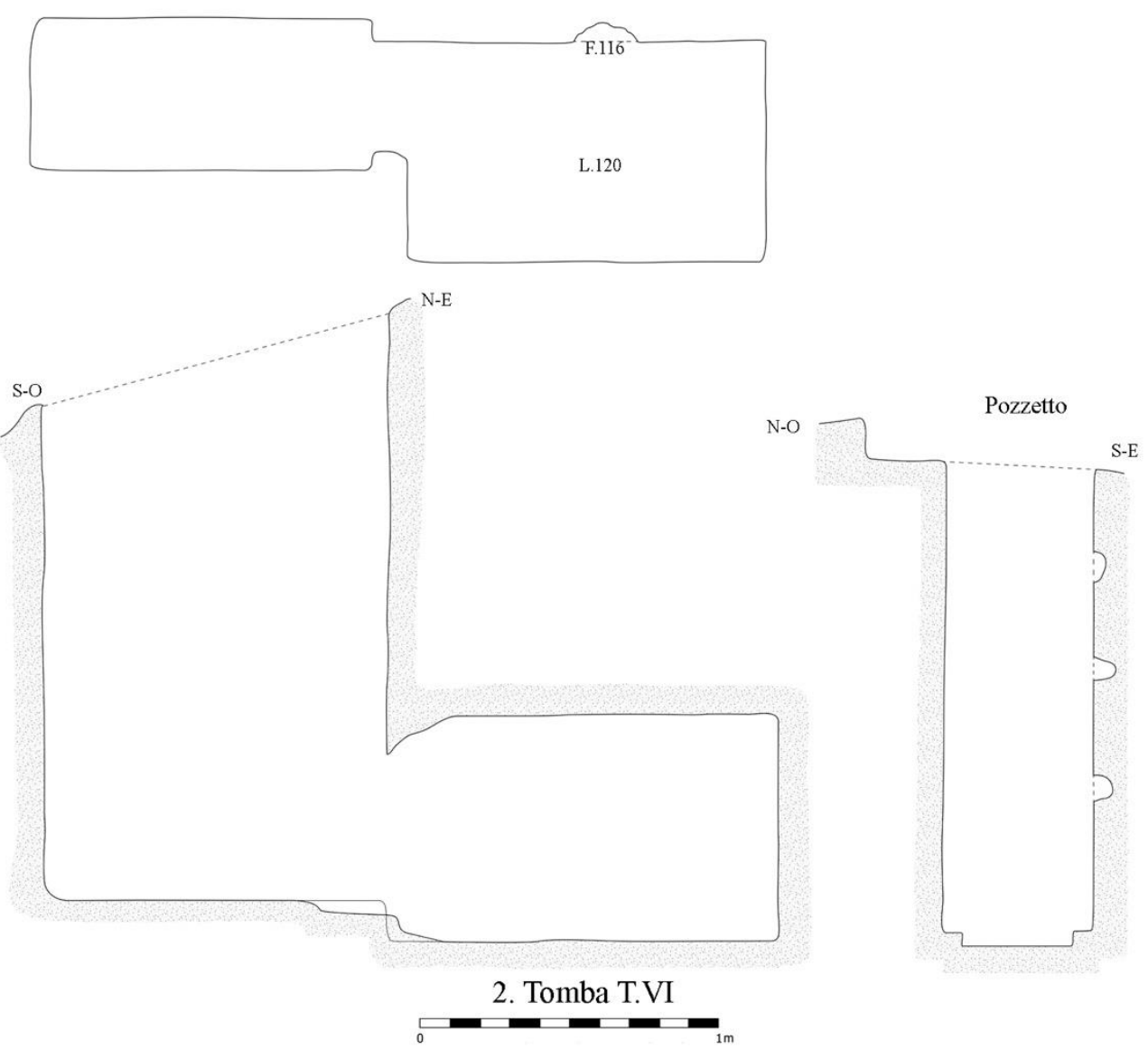

Fig. 8 - Nècropole - Dermech, Settore A: tombe a camera con accesso laterale, sezioni e pianta (scala 1.20). 

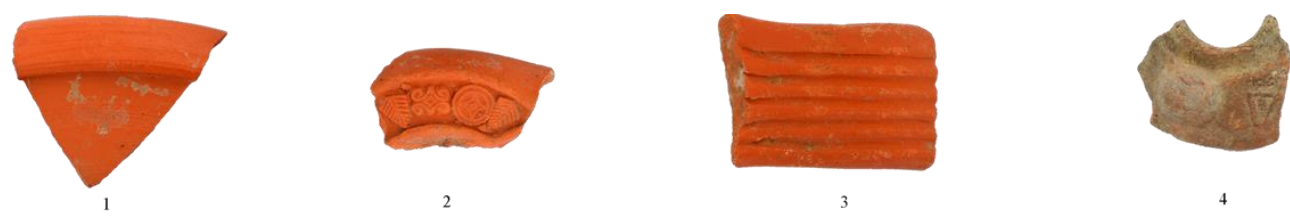

2
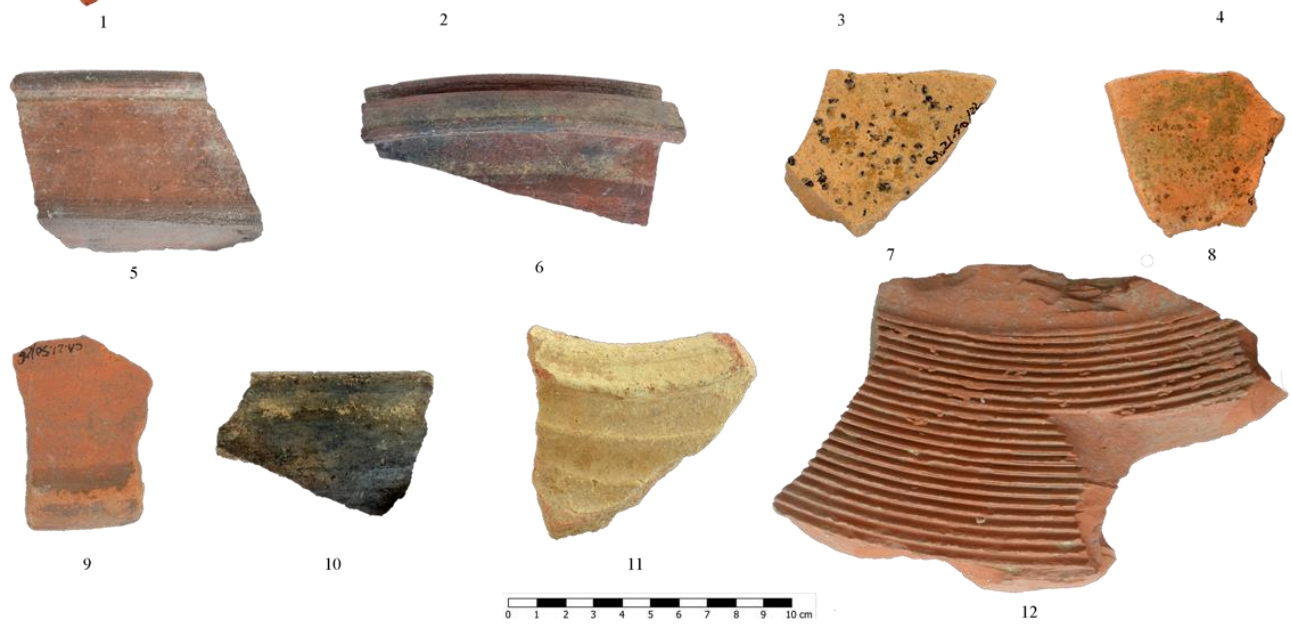

1

Fig. 9 - Selezione dei reperti ceramici rinvenuti all'interno del pozzetto verticale della tomba T.VI (US.50).
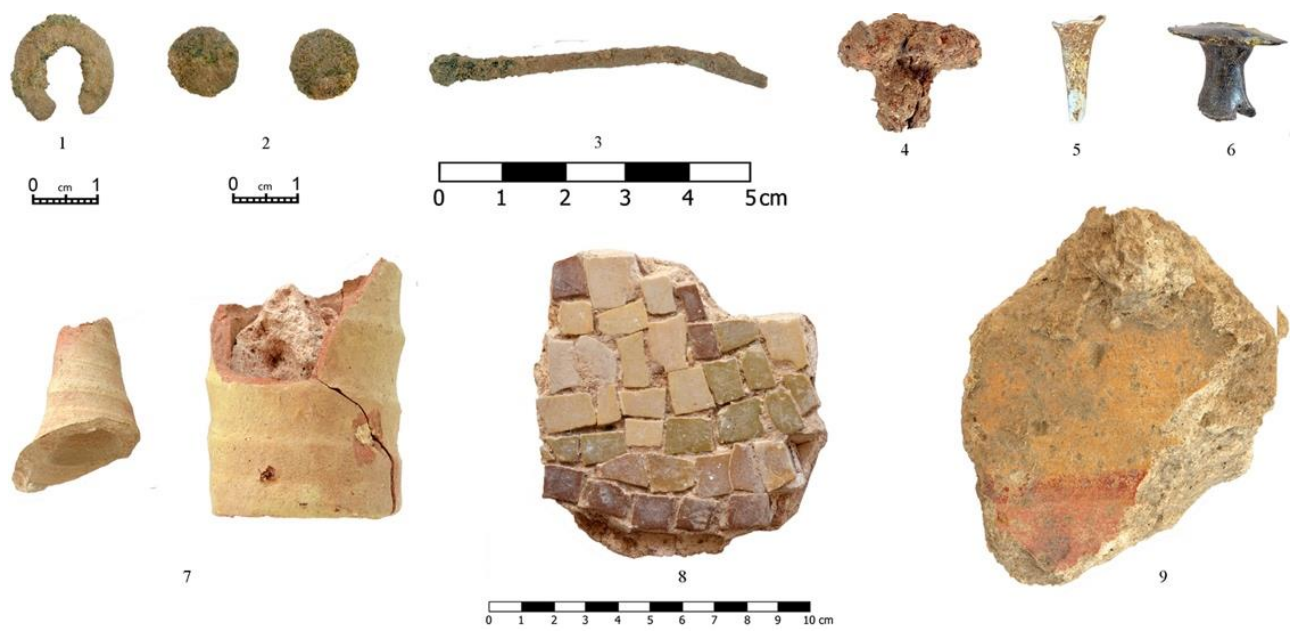

Fig. 10 - Selezione dei reperti rinvenuti all'interno della tomba T.VI: 1-5, 7-9) US.50; 6) US.112. 

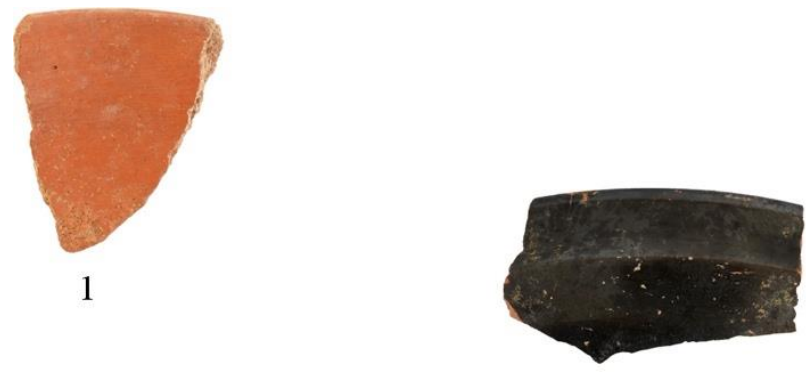

3

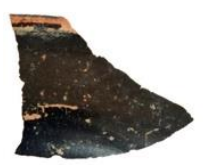

4

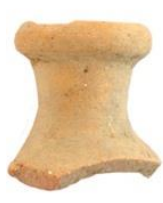

6

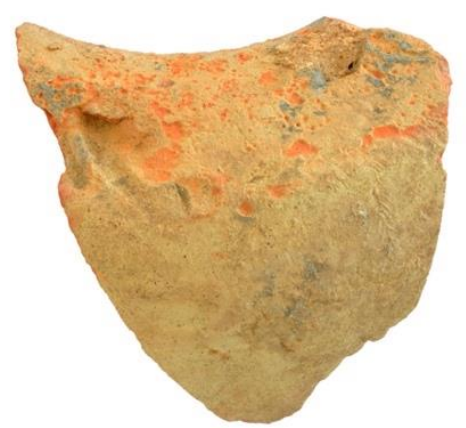

9

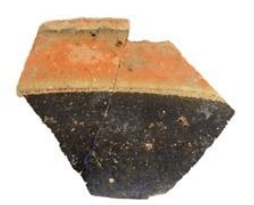

5

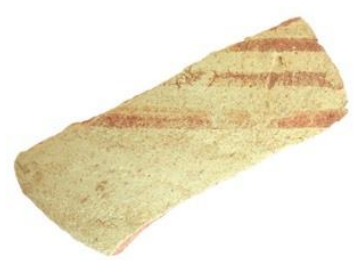

7

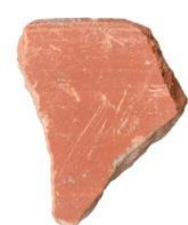

2

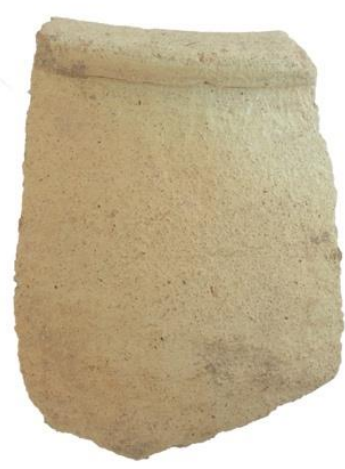

8

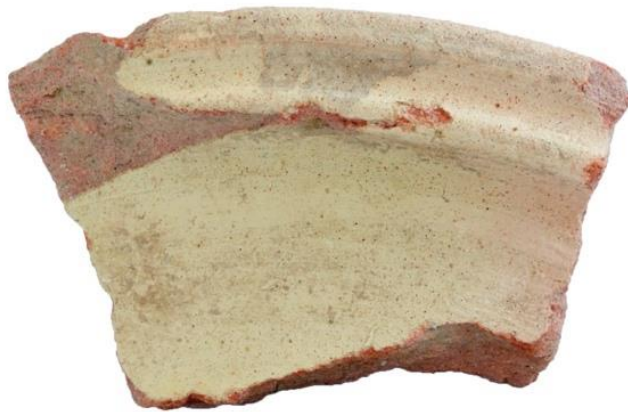

10

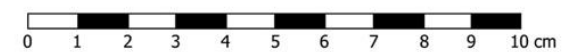

Fig. 11 - Selezione dei reperti ceramici dalla tomba T.VIII: 1) US.106; 2-10) US.54. 


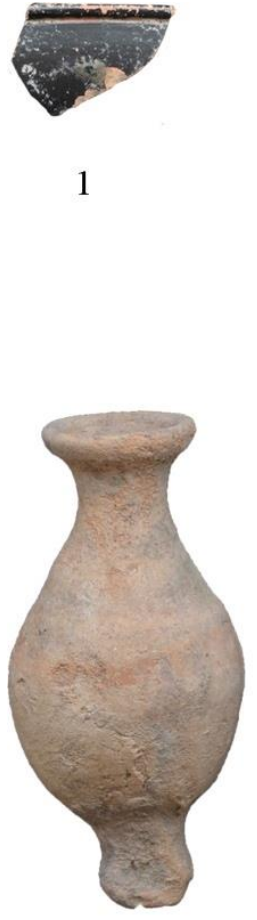

3

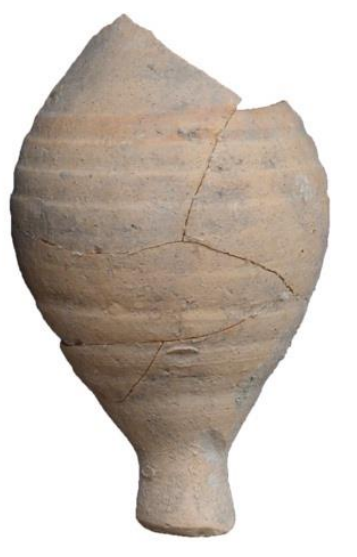

4

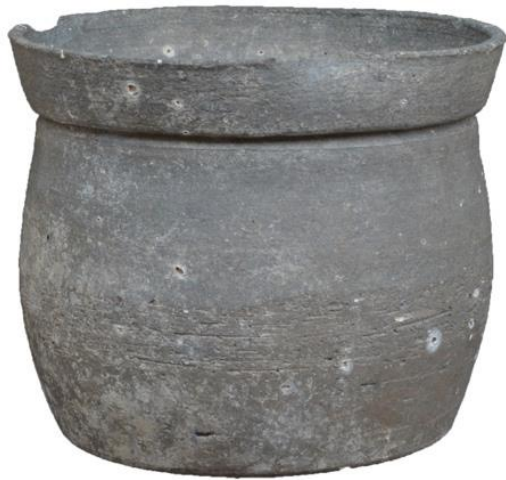

6

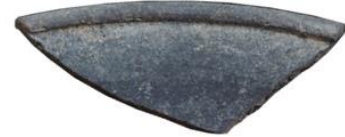

2

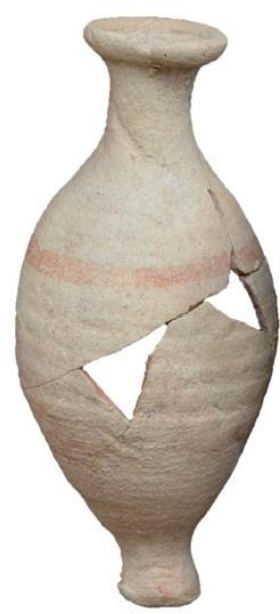

5

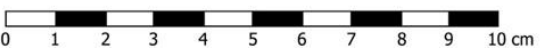

Fig. 12 - Reperti ceramici rinvenuti all'interno dello strato di crollo US.34 al di sopra della pavimentazione L.36 tra M.31 ed M.37 (Settore A). 

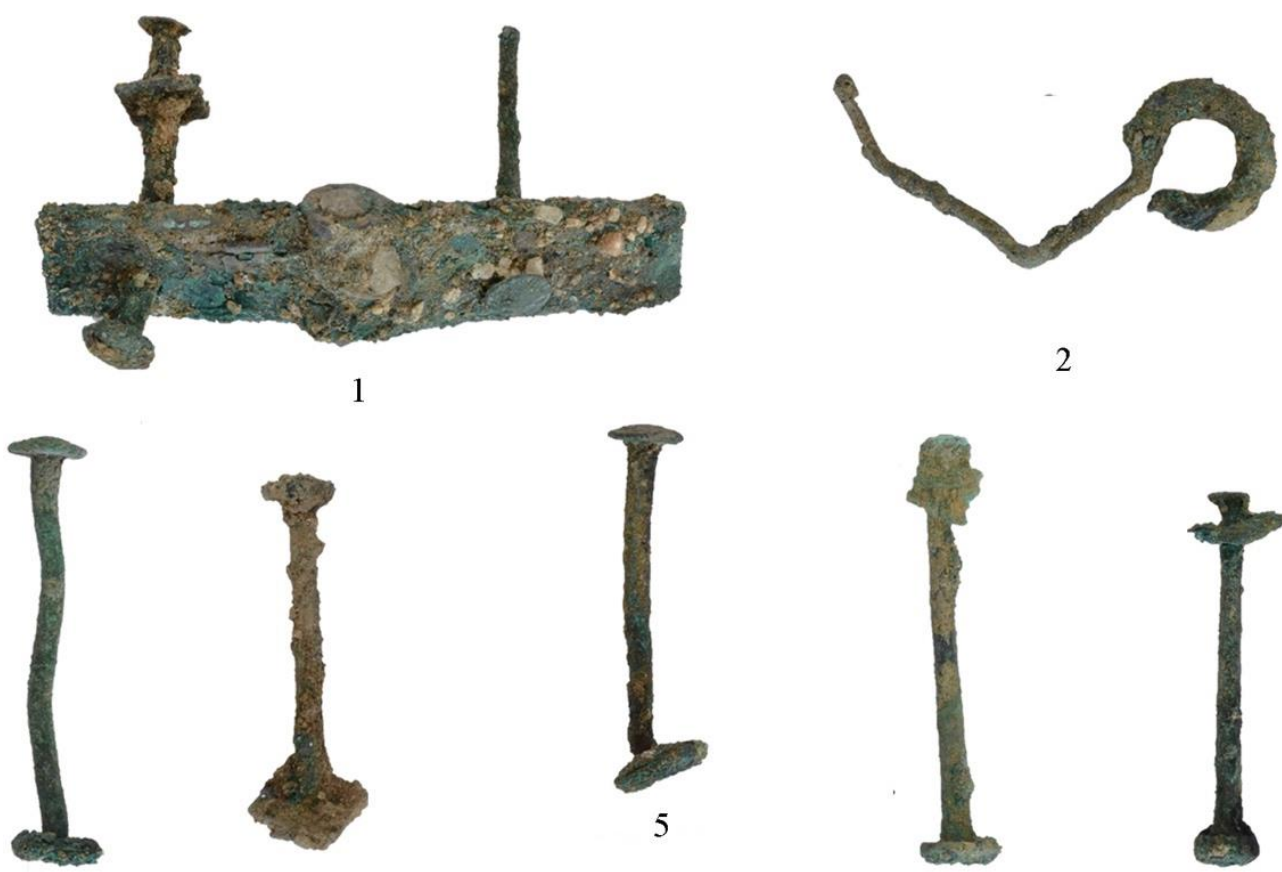

3

4

6

7

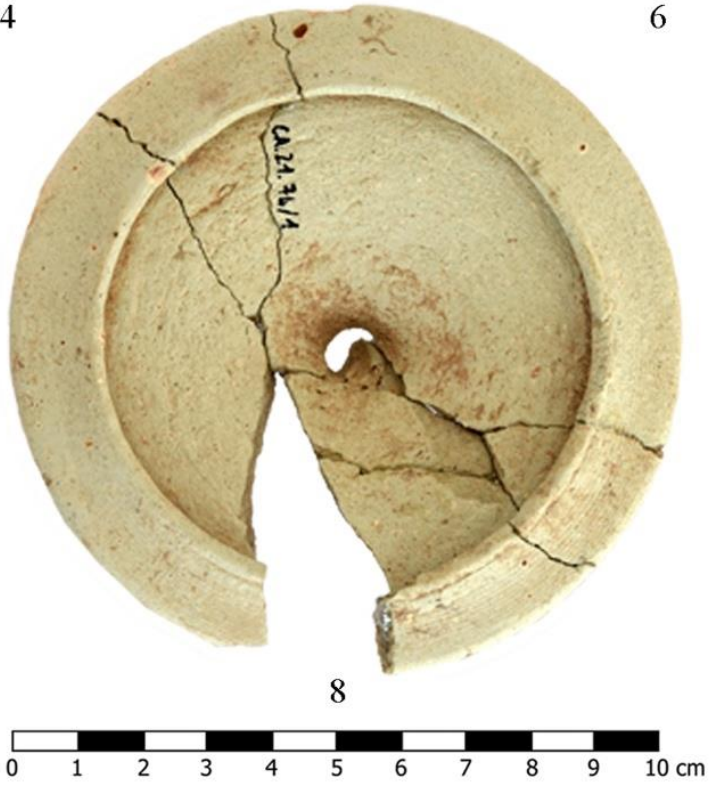

Fig. 13 - Reperti rinvenuti all'interno dello strato di crollo US.74 (Settore A, sezione sudoccidentale). 


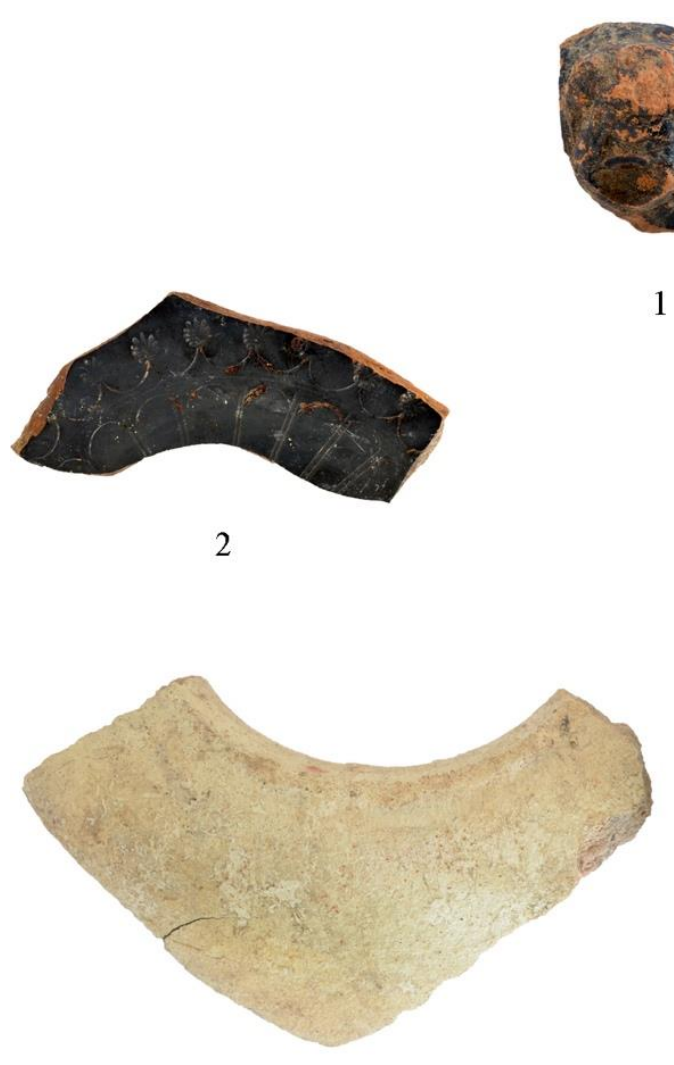

4

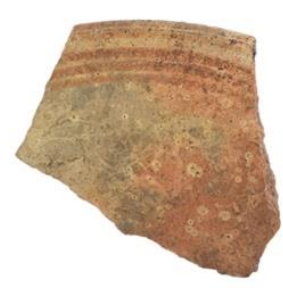

6

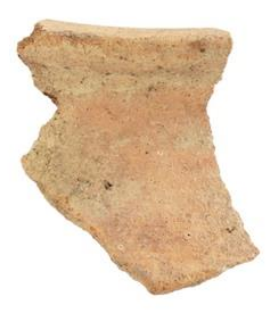

7

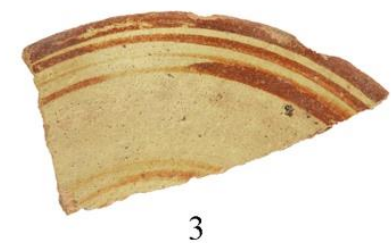

3

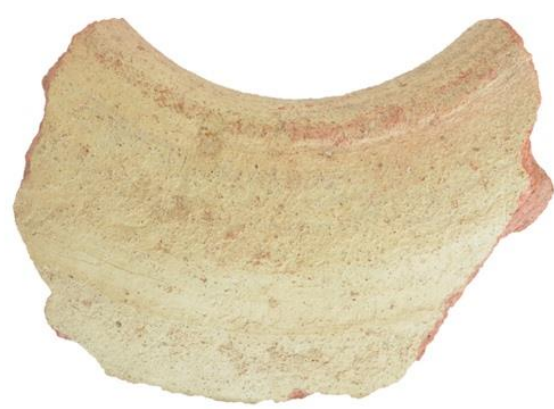

5

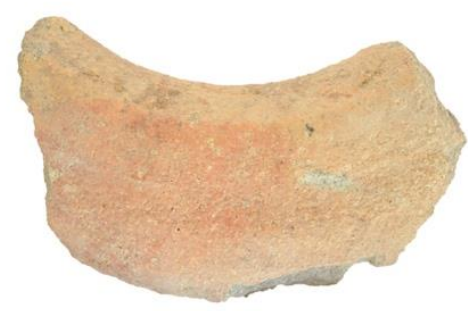

8

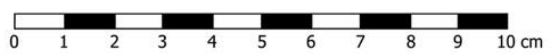

Fig. 14 - Selezione dei reperti ceramici rinvenuti all'interno degli strati US.78 (1), US.92 (2-5) e US.102 (6-8) (Settore A, sezione sud-occidentale). 


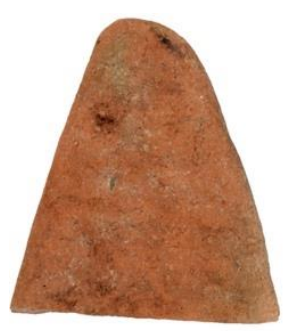

1

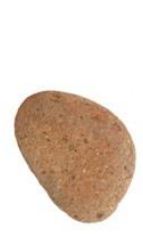

2

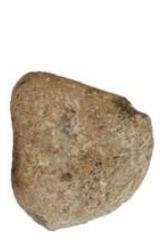

3

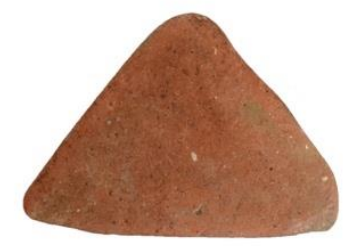

4

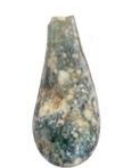

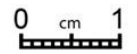

5

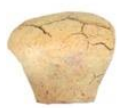

9

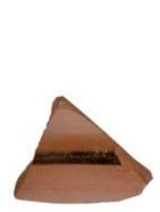

13

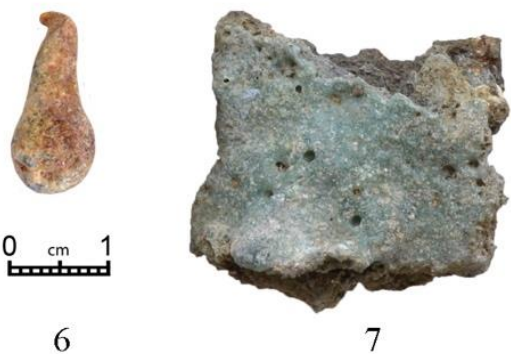

7

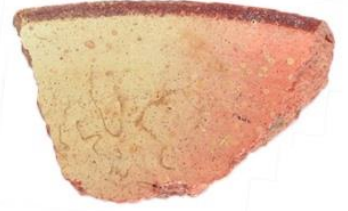

10

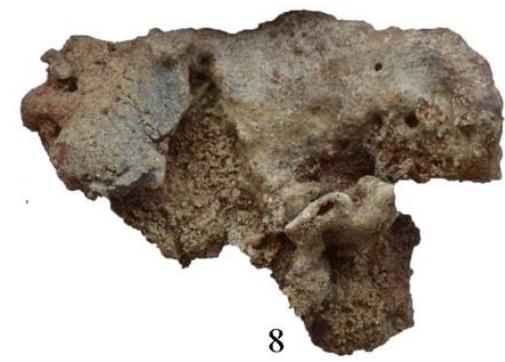

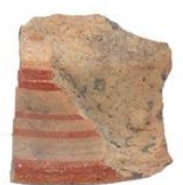

11

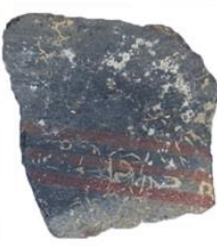

12

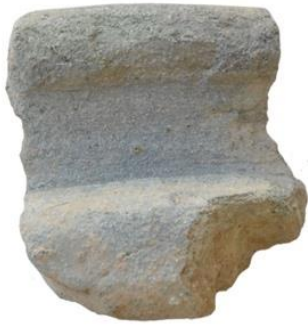

15

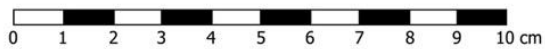

Fig. 15 - Selezione dei reperti rinvenuti all'interno degli strati US.94 (7-8; 9-12) e US.104 (1-6; 13-15) (Settore A, sezione sud-occidentale). 